\title{
AN INVESTIGATION OF RESTING STATE FUNCTIONAL CONNECTIVITY OF THE ANTERIOR AND POSTERIOR HIPPOCAMPAL SUBREGIONS IN POSTTRAUMATIC STRESS DISORDER AND ITS RELATIONSHIP WITH SYMPTOMS \\ by
}

\author{
Bailee L. Malivoire \\ Bachelor of Science, Brock University, 2015
}

\author{
A thesis \\ presented to Ryerson University \\ in partial fulfillment of the \\ requirements for the degree of \\ Master of Arts \\ in the program of \\ Psychology \\ Toronto, Ontario, Canada, 2017 \\ (C) Bailee L. Malivoire, 2017
}




\section{AUTHOR'S DECLARATION FOR ELECTRONIC SUBMISSION OF A THESIS}

I hereby declare that I am the sole author of this thesis. This is a true copy of the thesis, including any required final revisions, as accepted by my examiners.

I authorize Ryerson University to lend this thesis to other institutions or individuals for the purpose of scholarly research

I further authorize Ryerson University to reproduce this thesis by photocopying or by other means, in total or in part, at the request of other institutions or individuals for the purpose of scholarly research.

I understand that my thesis may be made electronically available to the public. 


\begin{abstract}
An Investigation of Resting State Functional Connectivity of the Anterior and Posterior Hippocampal Subregions in Posttraumatic Stress Disorder and its Relationship with Symptoms Master of Arts, 2017

Bailee L. Malivoire

Psychology

Ryerson University

Posttraumatic stress disorder (PTSD) is associated with abnormal hippocampal activity; however, the functional connectivity (FC) of the hippocampus with other brain regions and its relations with symptoms warrants further attention. I investigated FC of the hippocampus at a subregional level in PTSD during a resting state compared to trauma exposed controls (TECs). Based on imaging literature in PTSD, I targeted the FCs of the hippocampal head and tail subregions with the amygdala, medial prefrontal cortex (mPFC), and the posterior cingulate (PCC). The PTSD group had significantly greater FC compared to the TEC group between the left hippocampal head and the right amygdala, and for the left hippocampal tail with bilateral PCC. Resting state FC predicted symptom severity at time of scan and 4-months post-scan. These results highlight abnormal illness-related FC with both the hippocampal head and tail and provide support for future investigations of imaging biomarkers predictive of disease progression.
\end{abstract}




\section{Acknowledgements}

I would like to express my sincere appreciation to my advisor Dr. Todd Girard. Thank you for your unending support of my research goals and clinical pursuits, your patience in teaching me neuroimaging preprocessing and data analysis, and for sharing your immense knowledge in the field. I am extremely thankful to have a supervisor who is so invested in my research projects. I appreciate all your time, feedback, and ideas, which contributed to a stimulating and productive MA.

Thank you also to my thesis committee, Dr. Julia Spaniol and Dr. Candice Monson. Your respective expertise and contributions have been invaluable throughout the completion of this research project.

I would also like to thank Dr. Ronak Patel and members of the IMPACT lab for their integral role in data collection.

Lastly, I would like to thank my cohort for their unconditional support and encouragement. Thank you for always being there to lend an ear. 


\section{Table of Contents}

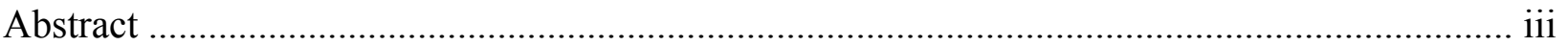

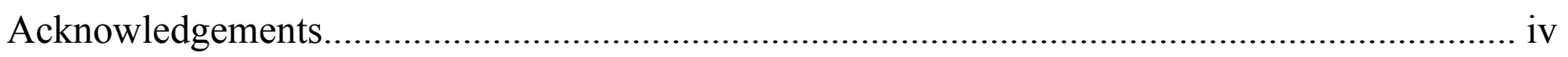

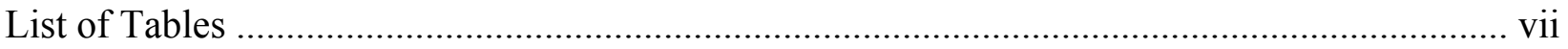

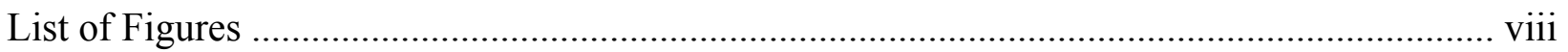

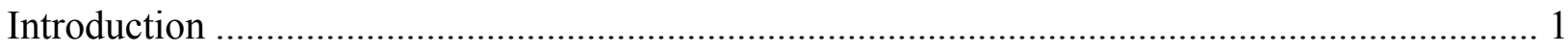

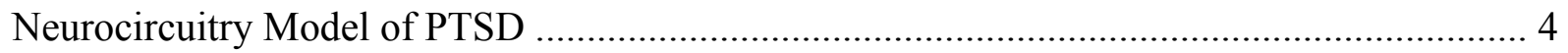

Anterior and Posterior Subdivisions of the Hippocampus ................................................ 5

fMRI Research Focusing on the Role of the Hippocampus in PTSD .................................. 6

Using rsFC to Investigate Hippocampal Abnormalities in PTSD .................................... 8

Resting State Functional Connectivity Research in PTSD ............................................. 8

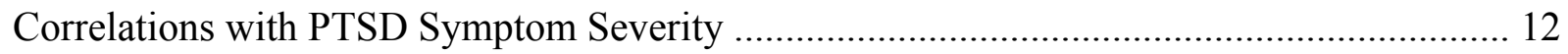

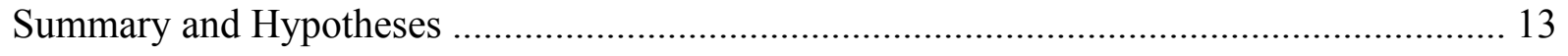

H1: The rsFC between the anterior hippocampus and the amygdala will differ between

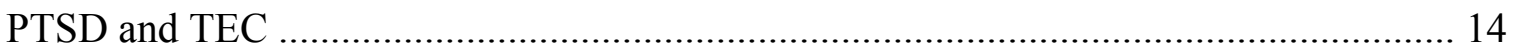

$\mathrm{H} 2$ : The rsFC between the anterior hippocampus and the mPFC will differ between PTSD

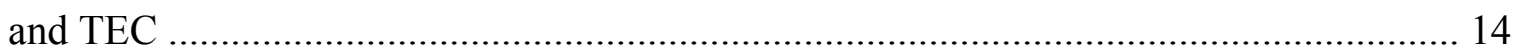

H3: The rsFC between the posterior hippocampus and the PCC will differ between PTSD

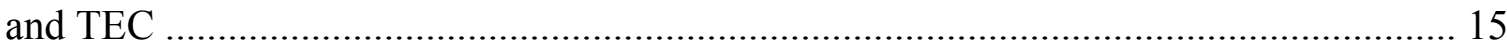

H4: Hippocampal rsFC will be associated with PTSD symptom severity at the time of scan and with PTSD symptom severity four months post-scan ................................ 15

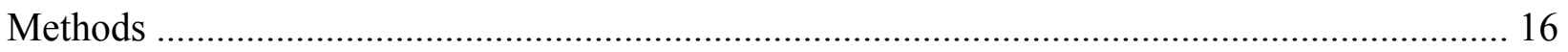

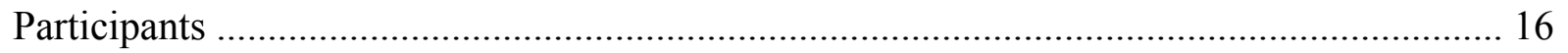

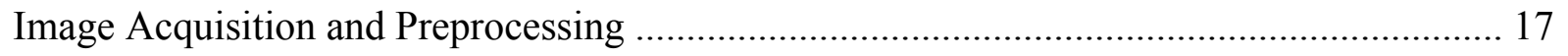




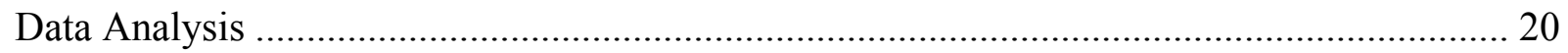

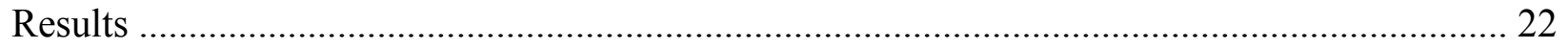

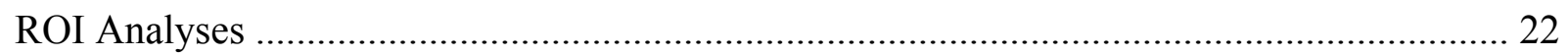

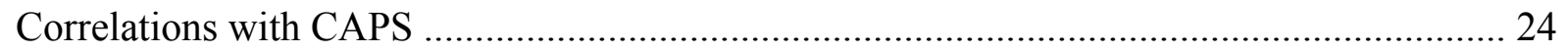

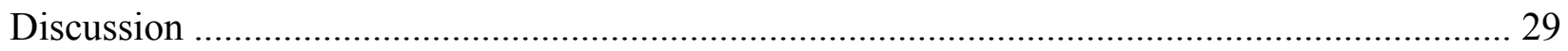

H1: The rsFC Between the Anterior Hippocampus and the Amygdala will Differ Between

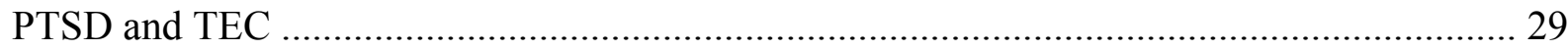

H2: The rsFC Between the Anterior Hippocampus and the mPFC will Differ Between PTSD

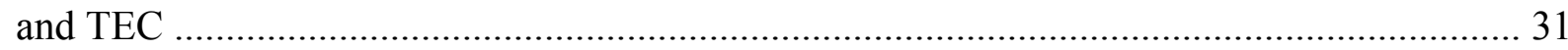

H3: The rsFC Between the Posterior Hippocampus and the PCC will Differ Between PTSD

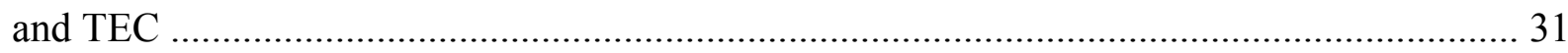

H4: Hippocampal rsFC will be Associated with PTSD Symptom Severity at the Time of Scan and with PTSD Symptom Severity Four Months Post-Scan ................................................ 33

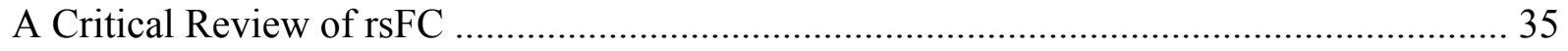

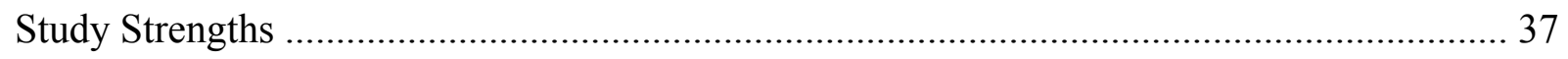

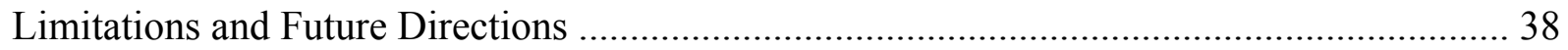

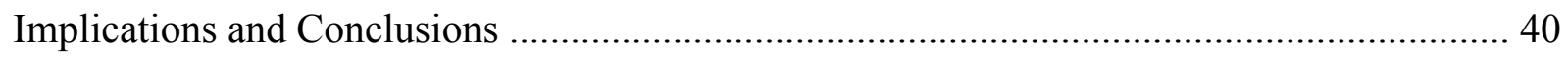

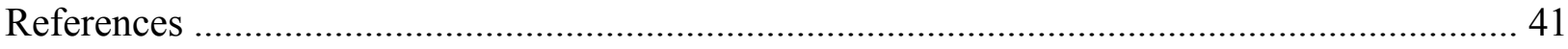




\section{List of Tables}

Table 1. Means and standard deviations for the FC coefficients with significant differences from the one- and two-sample t-tests identified 


\section{List of Figures}

Figure 1. The ROIs used in the present study displayed on a template brain.

Figure 2. The mean Fisher-z transformed FC values for the PTSD and TEC groups.

Figure 3. The association between CAPS scores at time of scan and the FC between the right hippocampal head and the left mPFC for the TEC and PTSD groups.

Figure 4. The association between CAPS scores at time of scan and the FC between the right hippocampal head and the right $\mathrm{mPFC}$ for the TEC and PTSD groups.

Figure 5. The association between CAPS scores at time of scan and the FC between the left hippocampal head and the right PCC for the TEC and PTSD groups.

Figure 6. The association between CAPS scores four month post scan and the FC between the right hippocampal head and the right amygdala for the TEC and PTSD groups.

Figure 7. The association between CAPS scores four month post scan and the FC between the left hippocampal head and the left PCC for the PTSD and TEC groups. 28

Figure 8. The association between CAPS scores four month post scan and the FC between the left hippocampal head and the right PCC for the PTSD and TEC groups. 


\section{Introduction}

Posttraumatic stress disorder (PTSD) is a debilitating psychiatric condition that develops consequent to trauma exposure, which includes direct exposure, witnessing trauma, learning that a close other was subjected to trauma, or through repeated, or intense, exposure to aversive details of a traumatic event (American Psychiatric Association, 2013). Symptoms commonly associated with PTSD can be grouped into categories of intrusions of the traumatic event (e.g., intrusive memories, flashbacks), hyperarousal in response to trauma-related cues, negative cognitions and mood, and withdrawal or avoidance behaviours (APA, 2013; Hughes \& Shin, 2011). Consequently, PTSD leads to significant impairment in daily functioning, including social dysfunction (Frueh, Turner, Beidel, \& Cahill, 2001; Solomon, 1989) and increased risk of suicide (Marshall et al., 2001). The symptoms associated with PTSD are proposed to be behavioural manifestations of changes in brain structure and function subsequent to trauma-associated stress (Bremner, 2006). Stress can lead to changes in brain structures, which ultimately result in alterations of brain circuits involved in the stress response (Bremner, 2006). Of those exposed to trauma, approximately 10\% develop PTSD (APA, 2013). Characterizing the neural basis of PTSD and the brain mechanisms underlying various PTSD symptoms is of interest to further advance our understanding of abnormal brain processes associated with the disorder and individual susceptibilities to the disorder. In addition, understanding abnormal brain functioning associated with a disorder can help identify possible biomarkers and advance treatment (Sripada et al., 2011; Zhou et al., 2012).

The goal of my thesis research is to help characterize the neural basis of PTSD by investigating resting-state functional connectivity (rsFC) using subregional hippocampal seed regions. As reviewed in more depth in subsequent sections, the hippocampus is a primary brain 
region in the proposed neurocircuitry underlying PTSD (Rauch, Shin, Whalen, \& Pitman 1998; Rauch, Shin, \& Phelps, 2006). Current theories of PTSD identify hippocampal dysfunction as a key contributor to hallmark symptoms of PTSD, including trauma-related intrusions in the form of flashbacks and nightmares, difficulty voluntarily recollecting trauma details, and overgeneralization of fear (APA, 2013; Carrion \& Wong, 2012). Moreover, people with PTSD demonstrate difficulty voluntarily recollecting particulars of the traumatic event (APA, 2013; Brewin, Gregory, Lipton, \& Burgess, 2010). In addition, persistent difficulties with everyday memory (Bremner, Vermetten, Afzal, \& Vythilingam, 2004; Roca \& Freeman, 2001; Uddo, Vasterling, Brailey, \& Sutker, 1993; Vasterling, Duke, Brailey, Constans, Allain, \& Sutker, 2002) and neuropsychological measures of working memory and episodic memory (Bremner et al., 1993; Yehuda, Keefe, Harvey, \& Levengood, 1995) have been identified in individuals with PTSD. However, the hippocampus has not been the focus of rsFC research in PTSD. To my knowledge, only one study has investigated subregional hippocampal rsFC in PTSD and the focus was a generalized anxiety disorder (GAD)-PTSD comparison (Chen \& Etkin, 2011). Moreover, hippocampal subregions have different structural and functional connections and contribute to different emotional and cognitive processes, as reviewed by Chase et al. (2015). Consequently, investigating hippocampal subregions independently may provide added insight into hippocampal FC in PTSD compared to trauma exposed controls (TEC). Moreover, the investigation of the rsFC of the subregions of the hippocampus and their relations with symptoms is a novel and important area of investigation in PTSD.

rsFC is a powerful tool used to investigate Blood Oxygenation Level Dependent (BOLD) signal changes at rest, which reflects local neuronal activity and is used to identify activated brain regions. There are several reasons why investigating rsFC using subregional hippocampal seed regions would improve our understanding of the role of the hippocampus in PTSD, as well as the 
neural basis of PTSD. First, there are discrepancies in the literature as to the direction and magnitude of abnormal activity of the hippocampus in PTSD (Astur et al., 2006; Geuze, Vermetten, Ruf, de Kloet, \& Westenberg, 2008; Jacques, Botzung, Miles, \& Rubin, 2011; Thomaes et al., 2009), and the discrepancies are proposed to be a consequence of the variety of tasks used (Hughes \& Shin, 2011; Patel, Spreng, Shin, \& Girard, 2012). rsFC is a powerful tool for investigating spontaneous activity of brain networks independently of a task and consequently could be used to help characterize abnormal intrinsic hippocampal FC. Secondly, rsFC studies avoid confounds inherent in task-based studies that complicate interpretations (Fornito \& Bullmore, 2010; Fox \& Greicius, 2010). For example, differences in performance on a task between a clinical population and controls could represent differences in approach, effort level, task performance, or an underlying brain abnormality that differentiates the two groups. In contrast, rsFC studies eliminate these ambiguities and permit investigation of fundamental abnormalities underlying a condition, such as PTSD (Fornito \& Bullmore, 2010; Fox \& Greicius, 2010). Furthermore, rsFC research provides insight into abnormal connectivity at rest, which is proposed to provide better insight into disease biomarkers and could serve as a potential diagnostic aid, given that the activation is not task-specific (Fornito \& Bullmore, 2010; Fox \& Greicius, 2010). Lastly, rsFC approaches improve the signal-to-noise ratio issue that arises in task-activation studies (Fox \& Greicius, 2010). Task-based activation studies use only $20 \%$ of the signal while the remaining $80 \%$ is unrelated to the task and considered noise. In contrast, ongoing spontaneous activity is the focus of rsFC studies, improving the signal-to-noise ratio by approximately three times (Fox \& Greicius, 2010). Thus, there is strong evidence in support of using rsFC methods to investigate hippocampal FC in PTSD.

The primary objectives of my thesis research included investigating rsFC with the anterior and posterior hippocampus as separate seed regions to evaluate their independent FCs. Following 
these analyses, I investigated whether the FC patterns were associated with PTSD symptom severity and whether they predicted PTSD symptom severity four months later. In the following sections I review the importance of the hippocampus within the context of PTSD symptoms and the neurocircuitry model of PTSD, I review task-based hippocampal region of interest (ROI) functional magnetic resonance imaging (fMRI) research in PTSD, rsFC research in PTSD, and research that has investigated the association between functional hippocampal activity and PTSD symptom severity. Moreover, the importance of investigating subregional hippocampal rsFC is highlighted and the advantages of rsFC analyses are discussed further.

\section{Neurocircuitry Model of PTSD}

The widely adopted neurocircuitry model of PTSD (Rauch et al., 1998, 2006) proposes that the amygdala, hippocampus, and medial prefrontal cortex (mPFC), which includes the anterior cingulate cortex (ACC) in this model, are dysfunctional in PTSD. Specifically, the amygdala is hypothesized to be hyperresponsive in response to threatening stimuli, which is proposed to contribute to intrusive trauma memories and hyperarousal (Rauch et al., 2006). The $\mathrm{mPFC}$ is suggested to have poor top-down control over the amygdala, which results in less amygdalar inhibition and may underlie deficits in attention and overgeneralized fear responses (Rauch et al., 2006). Reduced activation in the hippocampus is suggested to underlie impairments integrating contextual information about emotional memories and modulating amygdala activity

accordingly (Rauch et al., 1998, 2006). Since the development of the neurocircuitry model, research has supported that PTSD symptoms are associated with structural and functional changes in the interactions between the limbic system, including the amygdala, hippocampus, and the mPFC (Karl, Schaefer, Malta, Dörfel, Rohleder, \& Werner, 2006; Liberzon \& Sripada, 2007). 
Hippocampal connectivity and functioning are perturbed in PTSD more so than other anxiety-related disorders, such as GAD (Chen \& Etkin, 2013). Furthermore, connectivity between the hippocampus and amygdala in PTSD has been a focus in neuroimaging studies given that dysfunctional connectivity between these regions is proposed to underlie intrusion symptoms, which are hallmark symptoms in PTSD (Carrion \& Wong, 2012). In addition, the hippocampus is suggested to interact with the amygdala during encoding and contextualization of emotional memory. This connectivity is proposed to be dysfunctional in PTSD and to underlie core symptoms, such as hypervigilance (Carrion \& Wong, 2012). Consistent with the neurocircuitry model, some research supports the hypoactivity of the hippocampus and diminished hippocampal-amygdala connectivity (e.g., Sripada et al., 2012). However, inconsistent with the neurocircuitry model, other research suggests the hippocampus is hyperactive (e.g., Patel et al., 2012; Zhang, Zhang, Wang, Li, \& Zhang, 2016). This research will be further reviewed in subsequent sections. Investigating $\mathrm{rsFC}$ using other regions of the neurocircuitry model of PTSD, such as the amygdala and ACC, have provided insight into connectivity differences in PTSD compared to TEC. The hippocampus has yet to be used as a seed region in PTSD rsFC research compared to TEC. Moreover, hippocampal subregions have distinct connections to key brain regions in the neurocircuitry model and are proposed to underlie different PTSD memory-related symptoms (Carrion \& Wong, 2012; Chase et al., 2015). Thus, investigating subregional hippocampal $\mathrm{rsFC}$ will enhance our current understanding of the nature and function of the hippocampal connections within the neurocircuitry model and is the focus of my thesis.

\section{Anterior and Posterior Subdivisions of the Hippocampus}

Neuroimaging research supports a differentiation of function across anterior and posterior regions of the hippocampus (Chase et al., 2015). The posterior hippocampus is suggested to play 
a greater role in memory retrieval and spatial cognition and has connections with the pregenual ACC, the PCC, and the precuneus (Nadel, Hoscheidt, \& Ryan, 2013; Poppenk \& Moscovitch, 2011). Compared with the anterior hippocampus, the posterior hippocampus has been found to play a greater role in the default mode network, which is involved in integrating past events with the present stimuli and assessing the relevance to oneself (Chen \& Etkin, 2013). In contrast, the anterior hippocampus is more associated with the amygdala (Poppenk et al., 2013) and connected with the hypothalamic-pituitary-adrenal (HPA) axis and the limbic prefrontal circuitry (Chen \& Etkin, 2013; Fanselow \& Dong, 2010). In neuroimaging studies, fMRI activation of the anterior hippocampus is associated with emotional memory (Murty et al., 2010) and reward-directed behaviour (Viard et al., 2011). The anterior hippocampus specifically is hypothesized to underlie hippocampal-amygdala connections that contribute to atypical memory processes in PTSD, including flashbacks, intrusive thoughts, and nightmares (Carrion \& Wong, 2012) as well as overgeneralization of fear (Admon, Milad, \& Hendler, 2013). Thus, there is evidence to support differential roles of the anterior and posterior hippocampus in memories processes, which likely extends to PTSD symptoms. As summarized in the subsequent sections, neuroimaging research in PTSD to date has largely focused on treating the hippocampus as a homogeneous structure, which may contribute to discrepancies in the literature, such as in the direction of group differences in activation.

\section{fMRI Research Focusing on the Role of the Hippocampus in PTSD}

Neuroimaging research has investigated hippocampal activation in PTSD using a variety of memory tasks and symptom-eliciting tasks, as summarized below. Abnormal hippocampal grey matter volume and functioning in PTSD have been linked to deficits processing contextual information and memory impairments (Bonne et al., 2001; Shin et al., 2004; Werner et al., 2009). Functional neuroimaging studies that have focused on hippocampal responsivity to various tasks 
or symptom provocation in PTSD patients have obtained conflicting results. In fMRI research, patients with PTSD have reduced hippocampal activation during various memory tasks, such as the virtual Morris Water Maze task (Astur et al., 2006), retrieval of word-pairs (Geuze, Vermetten, Ruf, de Kloet, \& Westenberg, 2008), and a visuo-verbal working memory task (Moores et al., 2008). In contrast, other reports indicate patients with PTSD to have increased hippocampal activation during different memory tasks, such as encoding tasks (Thomaes et al., 2009), remembering word pairs (Werner et al., 2009), as well as in response to symptom provocation (Brohawn et al., 2010; Jacques et al., 2011). In sum, there is conflicting evidence as to the direction of differences in task-based hippocampal activation in PTSD.

Several explanations for the discrepancies have been proposed, including type of trauma exposure, type of comparison group, and differences in baseline activity (Patel et al., 2012). As discussed above, another likely contributing factor is the range of tasks used in different studies, which includes the actual experimental task participants are exposed to and the degree of arousal elicited using the probing stimuli (Hughes \& Shin, 2011; Patel et al., 2012). In addition, fMRI research has focused on the hippocampus as a single structure. The different structural and functional connectivities of hippocampal subregions could contribute to the discrepant findings. The bulk of research thus far has focused on patterns of brain activation in response to various tasks or while eliciting symptoms (Fox \& Greicius, 2010; Shin \& Liberzon, 2010). Only recently has research been conducted investigating patterns of brain connectivity at rest in PTSD (e.g., Sripada et al., 2011; Zhang et al., 2016), which explores FC more broadly and is commonly used to explore abnormal brain connectivity in psychiatric populations (Fox \& Greicius, 2010;

Greicius, 2008). Using a rsFC approach will permit investigation of hippocampal subregional FC that is not constrained to a particular task. rsFC avoids confounds inherent in task-based studies that complicate interpretations (e.g., differences in approach, effort-level) and is thought to yield 
more sensitive results compared to task-activation studies (Fornito \& Bullmore, 2010; Fox \& Greicius, 2010).

\section{Using rsFC to Investigate Subregional Hippocampal Abnormalities in PTSD}

rsFC explores synchronous activations between different brain regions at rest and is commonly used to explore how patterns of brain system connectivity in psychiatric conditions, such as PTSD, differ from controls (Fox \& Greicius, 2010; Greicius, 2008). Investigating abnormal brain activity using rsFC is proposed to have greater clinical utility compared to using a task-based approach as rs-fMRI investigates spontaneous activity of brain networks independently of a task and consequently characterizes abnormal intrinsic brain activity (Fox \& Greicius, 2010). Psychopathology is associated with disruptions in the organization of neural networks (Menon, 2011; van den Heuvel, Martijn, \& Hulshoff Pol, 2010). For instance, PTSD is proposed to manifest as a result of dysfunction in a functional network that encompasses the limbic system and cortical regions (Rabinak et al., 2011). Moreover, findings from a recent review in our lab (Patel et al., 2012) investigating PTSD neurocircuitry suggests that several memory-related PTSD symptoms (e.g., poor memory contextualization, unintentional retrieval of a trauma memory, etc.) are due to dysfunctions within brain networks, such as the default mode network and the salience network. Thus, researchers have begun investigating brain circuits and brain region interactions by quantifying the extent to which activity in one brain region is correlated with activity in other regions in PTSD. The following section summarizes the limited ROI-hippocampal rsFC research that has been conducted in PTSD to date.

\section{Resting State Functional Connectivity Research in PTSD}

Given the hypothesized importance of the amygdala underlying PTSD symptoms, such overgeneralization of fear and exaggerated startle response (Carrion \& Wong, 2012), the amygdala has been a primary ROI in rsFC investigations in PTSD (Rabinak et al., 2011; Sripada 
et al., 2011; Zhang et al., 2016). Rabinak et al. (2011) conducted one of the first amygdala rsFC studies in male PTSD veterans compared to a TEC group, which consisted of combat-exposed male veterans without PTSD. By correlating the average time course of activity in the left and right amygdala seed regions with all other voxels in the brain, Rabinak et al. (2011) found stronger connectivity between the right amygdala and the insula for the PTSD group compared to controls. There were no group differences in connectivity between the amygdala and other predicted regions, including the hippocampus (Rabinak et al., 2011). Sripada et al. (2011) investigated amygdala rsFC in male veterans compared to a male TEC sample using globalsignal regression procedures. There was greater positive connectivity between the right amygdala and left hippocampus in the TEC group compared to the PTSD group. Using similar methods as Sripada et al. (2011), Zhang et al. (2016) investigated differences in amygdala rsFC between a PTSD group and a TEC group. One of the group differences in FC was between the amygdala and hippocampus. Specifically, greater connectivity was found between the amygdala and hippocampus for the PTSD group compared to controls. Thus, Rabinak et al. (2011) did not find resting state amygdala-hippocampal FC differences when comparing the PTSD group with controls, while Sripada et al. (2011) found reduced amygdala-hippocampal connectivity in the PTSD group and Zhang et al. (2016) found greater connectivity in the PTSD compared to TEC. These inconsistencies warrant further research to clarify the direction of differences in amygdalahippocampal rsFC between PTSD and TEC. The aforementioned rsFC studies treated the hippocampus as a homogeneous structure. However, the hippocampal subregions have different functional connections and contribute to different memory processes, which may be relevant to PTSD symptoms (Admon, Milad, \& Hendler, 2013; Carrion \& Wong, 2012; Poppenk et al., 2013). Using subregional hippocampal seed regions could help clarify our understanding of the direction of differences in amygdala-hippocampal rsFC in PTSD relative to TEC. 
Additional research that has investigated rsFC in PTSD has used the posterior cingulate cortex (PCC), ACC, and thalamus as seed regions for connectivity analyses. Using independent component analysis, there was weaker connectivity between the PCC seed region and the right hippocampus, right amygdala, and right insula in the PTSD group compared to controls (Bluhm et al., 2009). The PCC is part of the default mode network and thus differences between PTSD and non-trauma controls in medial temporal lobe-PCC connectivity may contribute to improper integration and contextualization of memories, which may contribute to being hypersensitive in situations that are not relevant to the trauma and do not pose a threat (Bluhm et al., 2009). Using whole-brain FC, Jin et al. (2014) found weaker positive connectivity for those with PTSD compared to TEC between the MPFC and the limbic system, specifically both the amygdala and hippocampus/parahippocampal gyrus. Group differences in connectivity between the mPFC and the hippocampus may be associated with differences in inhibition of negative, trauma-related memories (Jin et al., 2014). Although other seed regions have been used to investigate FC, group differences in connectivity were not found between the hippocampus and seeds in the thalamus (Yin et al., 2011) or the ACC (Kennis et al., 2015). It is also evident that studies implicate more brain regions than those captured by the traditional neurocircuitry model in PTSD, which is attributable to recent advances in our understanding of neurocircuitry in PTSD (Patel et al., 2012).

Despite differences in the function of the anterior and posterior hippocampal subregions, few studies have investigated subregional hippocampal rsFC. One recent exception is a study by Chen and Etkins (2013) that investigated anterior and posterior hippocampal rsFC differences between patients with PTSD and GAD. Compared to GAD, the PTSD group had diminished connectivity between the posterior hippocampus and the areas of interest, which suggests posterior hippocampal connectivity is perturbed in PTSD more so than GAD. Specifically, 
compared to both GAD and controls, connectivity in the PTSD group was diminished between the posterior hippocampus and brain regions part of the default mode network, including the PCC and pregenual ACC (Chen \& Etkin, 2013). Connectivity between the posterior hippocampus and the precuneus, another region in the default mode network, was perturbed when the PTSD group was compared to healthy controls only (Chen \& Etkin, 2013). There were no differences between the PTSD and GAD groups in their anterior hippocampal connectivity as both groups significantly differed from controls in their connectivity with the dorsal ACC/ pre-supplementary motor area (Chen \& Etkin, 2013). Due to the different hypothesized functions of the anterior and posterior hippocampus and the findings of Chen and Etkin (2013) that support different subregional hippocampal connectivity in PTSD compared to GAD, further investigations of subregional hippocampal rsFCs are warranted. Chen and Etkin (2013) acknowledge the lack of a TEC group as a limitation relevant to the PTSD group as this comparison would elucidate abnormal connectivity differences in individuals exposed to trauma who developed PTSD compared to those who did not subsequently develop the disorder. Addressing this limitation, my thesis includes a TEC group for comparison.

In summary, from the existing rsFC studies in PTSD it is evident that there are group differences in connectivity using whole brain FC analyses (Jin et al., 2014) as well as using seed ROI analyses (e.g., Bluhm et al., 2009; Spripada et al., 2011). Specifically, rsFC differences between PTSD and controls have been observed between the hippocampus and the amygdala (Sripada et al., 2011; Zhang et al., 2016), the PCC (Bluhm et al., 2009), and the mPFC (Jin et al., 2014). However, limited research has been conducted investigating rsFC between these ROIs and subregional hippocampal seed regions. One exception is the research study by Chen and Etkins (2013) that focused on rsFC differences between GAD and PTSD and did not include a TEC group. Thus, the present study will make an important contribution to the literature by 
investigating subregional hippocampal rsFC in PTSD compared to TEC, which will elucidate FC differences in those exposed to trauma who develop PTSD and those who are exposed to trauma but who do not go on to develop the disorder. Investigating FC between hippocampal subregions and key brain regions within brain networks, such as the amygdala, will refine our understanding of hippocampal-dysfunction in PTSD. Moreover, investigating hippocampal connectivity with the mPFC and the PCC will help characterize its abnormal connections within the default mode network (Patel et al., 2012).

\section{Correlations with PTSD Symptom Severity}

Research has also investigated how brain activation and rsFC relate to PTSD symptoms as measured by the Clinician Administered PTSD Scale (CAPS; Blake et al., 1995). CAPS is a commonly used and well-validated measure of PTSD symptom severity that is frequently used to assist clinicians when diagnosing PTSD (Blake et al., 1995). Hippocampal activation has been found to be negatively correlated with PTSD symptom severity using a variety of memory tasks, such as the virtual Morris Water Maze task (Astur et al., 2006), an explicit memory task (Shin et al., 2004), encoding of trauma-related stimuli (Hayes et al., 2011), and at rest (Marin et al., 2016). rsFC techniques have also been used to explore whether patterns of FC are associated with symptom severity in PTSD. For instance, PCC/precuneus connectivity with the amygdala in PTSD patients is positively associated with CAPS scores and predictive of future symptoms six weeks later (Lanius et al., 2010). Connectivity between the PCC and left mPFC is negatively correlated with CAPS scores (Quin et al., 2012). Moreover, PCC connectivity with the right hippocampus, right amygdala, and left superior temporal gyrus are negatively correlated with CAPS scores (Zhou et al., 2012). The strength of the connectivity between the PCC and amygdala predict PTSD symptom severity one to six months following the traumatic event (Zhou et al., 2012). Thus, there is evidence to suggest that characteristics of FC are associated with 
PTSD symptom severity and can predict PTSD symptom severity at a later time. Investigating relationships between FC and PTSD symptom severity can help to determine possible biomarkers of PTSD or identify individuals with a greater predisposition for the disorder (Zhou et al., 2012). The hippocampus is a central structure in the neurocircuitry model of PTSD (Rauch et al., 2006) and abnormal hippocampal connectivity is suggested to underlie key symptoms in PTSD (Carrion \& Wong, 2012). Consequently, hippocampal connectivity in PTSD may be predictive of PTSD symptom severity and could be a biomarker of the disorder; however this has yet to be investigated directly. In my thesis I assess the relationship between hippocampal functional connectivity and PTSD symptoms.

\section{Summary and Hypotheses}

Current theories of PTSD identify hippocampal dysfunction as a key contributor to hallmark symptoms of PTSD including trauma-related intrusions in the form of flashbacks, intrusive thoughts, and nightmares, difficulty voluntarily recollecting trauma details, and overgeneralization of fear (APA, 2013; Brewin et al., 2010; Carrion \& Wong, 2012). To my knowledge, research has yet to use subregional hippocampal seed regions during a rsFC analysis comparing PTSD to a TEC group. Discrepancies in previous findings may be a consequence of treating the hippocampus as a single structure. A more nuanced understanding of hippocampal connectivity may result from investigating hippocampal subregions independently. Specifically, these analyses may clarify the direction of hippocampal-amygdala connectivity and will build on previous reports of differences in hippocampal FC between PTSD and controls (e.g., Bluhm et al., 2009; Jin et al., 2014). Moreover, given the integral role of the hippocampal in PTSD symptoms, subregional hippocampal rsFC is a likely predictor of PTSD symptom severity; however, this has yet to be investigated. 
For my Master's thesis, I investigated rsFC in PTSD using anterior and posterior hippocampal seed regions. Based on imaging literature, I made a priori hypotheses and investigated the rsFCs of the anterior and posterior hippocampal subregions with the amygdala, mPFC, and the PCC. Furthermore, I investigated whether group rsFC is associated with PTSD symptom severity and whether it is predictive of PTSD symptoms four months later. Patients with PTSD were compared to TECs. Four hypotheses were made based on previous findings as follows:

\section{H1: The rsFC between the anterior hippocampus and the amygdala will differ}

between PTSD and TEC. Dysfunction in hippocampal-amygdala connectivity is proposed to be associated with hallmark symptoms of PTSD, including intrusive thoughts and flashbacks (Carrion \& Wong, 2012). There are differences between PTSD and control groups in amygdalahippocampal connectivity using the amygdala as a seed region (Sripada et al., 2011; Zhang et al., 2016). Consequently, it was hypothesized that I would find differences between the PTSD and TEC groups in rsFC between the hippocampus and amygdala using the hippocampus as a seed region. Specifically, rsFC between the PTSD and TEC groups is expected to differ for the anterior hippocampus and the amygdala given its proposed association with the amygdala (Poppenk et al., 2013). Given the conflicting evidence as to the directionality of the rsFC between the amygdala and hippocampus in PTSD compared to TEC (Rabinak et al., 2011; Sripada et al., 2011; Zhang et al., 2016), a directional hypothesis was not made.

\section{H2: The rsFC between the anterior hippocampus and the mPFC will differ between}

PTSD and TEC. The mPFC has been found to have strong connections with the hippocampus, both regions are part of the neurocircuitry model of PTSD, and Jin et al. (2014) found there to be abnormal connectivity between the mPFC and the hippocampus in a whole-brain FC analysis. In rodents, connectivity between the PFC and hippocampus originates in the ventral CA1 and 
subiculum (Li, Long, \& Yang, 2015). Limited data regarding subregional hippocampal-prefrontal cortex connectivity is available in humans ( $\mathrm{Li}$, et al., 2015); however, the findings in rodents would suggest the connectivity between the hippocampus and PFC stems from the anterior hippocampus. Thus, I hypothesized that I would find group differences in $\mathrm{rsFC}$ between the anterior hippocampus and $\mathrm{mPFC}$.

H3: The rsFC between the posterior hippocampus and the PCC will differ between PTSD and TEC. Past research has also found reduced connectivity between the PCC and the hippocampus in PTSD (e.g. Bluhm et al., 2009). Furthermore, Chen and Etkin (2013) found the posterior hippocampus to have perturbed connectivity with the default mode network, which included the PCC, when comparing PTSD to GAD. Thus, differences between PTSD and TEC are predicted for rsFC between the posterior hippocampus and the PCC.

H4: Hippocampal rsFC will be associated with PTSD symptom severity at the time of scan and with PTSD symptom severity four months post-scan. Given evidence supporting the association between hippocampal activation and PTSD symptom severity (Astur et al., 2006; Hayes et al., 2011; Marin et al., 2016) and that rsFC between the PCC and medial temporal lobe is negatively correlated with PTSD symptom severity (Zhou et al., 2012), it is hypothesized that rsFCs with the hippocampus will also be associated with PTSD symptoms. Given research has also found rsFC to predict future PTSD symptoms (Lanius et al., 2010), subregional hippocampal rsFC was predicted to be associated with symptoms four months post-scan. 


\section{Method}

\section{Participants}

Resting-state data were analyzed from participants who took part in a previous task-based fMRI study that comprised recently traumatized individuals $(N=24$; Patel et al., 2016). Participants were recruited from two studies conducted by the Investigating Methods to Prevent, Assess, and Care for Trauma (IMPACT) lab at Ryerson University. Eighteen participants were recruited from a study investigating PTSD symptoms within a trauma-exposed sample over time while the remaining six participants were recruited from a PTSD couple's therapy research study. At the time of scan, 11 participants met criteria for PTSD while the remaining 13 were TECs. CAPS was used to assess for PTSD and has been demonstrated to have excellent reliability and validity for assessment of PTSD symptoms (Blake et al., 1995). A CAPS score greater than 45 was required for a diagnosis of PTSD. In addition, consistent with DSM-IV criteria, the participants needed to endorse the presence of at least one re-experiencing symptom, three numbing or avoidance symptoms, and two hyperarousal symptoms. Items on the CAPS were considered to meet diagnostic threshold if the participant was assigned a score of at least one on frequency and two on intensity within the past month. CAPS was administered at the time of scan and four months post scan. In the PTSD group, the mean age was 34 (range $=19-55$ ) and five of the 11 participants were male. The PTSD group was exposed to a variety of traumatic events including sexual assault $(n=5)$, physical assault and/or death threats $(n=4)$, witnessing a violent physical assault or death $(n=1)$, and combat exposure $(n=1)$. Approximately half of the participants within the PTSD group were exposed to their trauma within 1 year prior to the scan $(n=6)$. The requirements for the TEC group were exposure to a traumatic event in accordance with criterion A of the CAPS and that they had a total CAPS score of less than 30 at time of scan. 
In the TEC group, the mean age was 32 (range $=18-52$ ) and four of the 13 participants were male. The types of trauma exposure varied within the TEC group and included motor vehicle or biking accident $(n=5)$, witnessing a violent assault and/or death threats $(n=4)$, and direct exposure to physical assault or death $(n=4)$. All but two participants in the TEC group had been exposed to their trauma within one year before the scan.

Psychological comorbidities were assessed using either the Mini International Neuropsychiatric Interview ( $n=18$; Sheehan et al., 1997) or the Structured Clinical Interview for DSM-IV ( $n=6$; First, Spitzer, Gibbon, \& Williams, 2002) depending on study recruitment. There were several comorbid diagnoses within the PTSD group including anxiety $(n=6)$, major depression $(n=4)$, or substance use disorder $(n=2)$. Five of the 11 PTSD patients were taking anti-depressants at the time of assessment. With regards to the TEC group, only two participants had a comorbid anxiety disorder, one had both alcohol abuse and substance dependence, and two participants were taking medication. The Beck Depression Inventory-II (BDI-II; Beck, Steer, \& Brown, 1996) was used to assess depressive symptoms.

\section{Image Acquisition and Preprocessing}

Anatomical and resting-state fMRI images were acquired in an axial orientation using a 3T Sigma MR System (GE Medical Systems, Milwaukee), located at Toronto Western Hospital, as part of a previous fMRI study from our lab (Patel et al., 2016). $\mathrm{T}_{1}$-weighted anatomical scans were acquired using a fast spoiled gradient echo (FSPGR) sequence at a resolution of isotropic 1$\mathrm{mm}^{3}$ voxels (176 slices, $256-\mathrm{mm}$ FOV, $256 \times 256$ matrix). As outlined below, these $\mathrm{T}_{1}$ scans were used to co-register the resting-state functional scans, derive tissue-segmented maps, and determine normalization parameters. Resting-state data were collected for $308 \mathrm{~s}(\mathrm{TR}=2000 \mathrm{~ms}$, $\mathrm{TE}=30 \mathrm{~ms}$, flip angle $=85^{\circ}, 40$ slices) at a spatial resolution of $3.125 \times 3.125 \times 4.0 \mathrm{~mm}^{3}$ voxels $(\mathrm{FOV}=200 \mathrm{~mm}$ ). During the resting-state scan, participants laid face upward with their eyes 
open while viewing a black fixation cross on a white screen for five minutes. The resting state scans were preprocessed using the Data Processing \& Analysis of Brain Imaging (DPABI) toolbox (Yan, Wang, Zuo, \& Zang, 2016), which ran on MATLAB version 7.10.0 (The MathWorks, Natick, MA). DPABI includes Data Processing Assistant for Resting-State fMRI Advanced (DPARSFAv3.2, by Yan Chao-Gan, http:// www.restfmri.net), and is updated from the Resting-State fMRI Data Analysis Toolkit (REST; Song et al. 2011). Each participant's resting state scan consisted of 154 time points. The first 10 time points were removed for each participant for signal equilibrium and the remaining 144 images were preprocessed. The images were corrected for slice timing and a Friston 24-parameter head motion correction was applied, which regresses out head motion effects from the realigned images (Friston, Williams, Howard, Frackowiak, \& Turner, 1996). In addition, nuisance covariates including white matter and cerebrospinal fluid were regressed. White matter and cerebrospinal fluid masks were generated from each participant's segmentation map using a probability threshold of 0.99 . The nuisance covariates were regressed from the time series using the CompCor method (Behzadi et al. 2007), which creates a combined white matter/cerebrospinal fluid mask and extracts the first five principal components to reduce noise related to respiratory and cardiac effects. Time points with too much motion were defined as volumes with FD (Jenkinson) $>0.2 \mathrm{~mm}$ and volumes two forward one back from these volumes (Jenkinson, Bannister, Brady, \& Smith, 2002), which is consistent with recommended DPABI methods (Yan, Wang, Zuo, \& Zang, 2016). Times points with motion above this threshold were regressed out.

The DARTEL tool (Ashburner, 2007) was used to normalize the functional images into a standard stereotaxic anatomical Montreal Neurological Institute (MNI) space. The normalized volumes were resampled to a voxel size of $3 \mathrm{~mm} \times 3 \mathrm{~mm} \times 3 \mathrm{~mm}$. This process involved three steps including coregistration, segmentation, and writing normalization parameters. The images 
were smoothed with a 4-mm full-width, half-maximum isotropic Gaussian kernel, which is consistent with similar reports in the PTSD rsFC literature (e.g., Zhou et al., 2012). Temporal filtering $(0.01-0.1 \mathrm{~Hz})$ was applied following smoothing to the time series of each voxel to reduce the effect of low and high frequency noise. Using the quality control feature of DPABI the accuracy of the coregistration, segmentation, and normalization of the images were checked. The head motion metrics were also reviewed for each participant. Lastly, a mean regression and standard deviation division standardization module was applied, which is recommended to reduce the impact of nuisance variation on resting state fMRI measures (Yan, Wang, Zuo, \& Zang, 2016).

Hypothesis-driven ROI-based analyses were used, as opposed to an exploratory wholebrain approach, given the study aims. This approach also mitigates the severity of correction for multiple tests and was deemed more appropriate for the current sample size. Based on a priori hypotheses, separate ROIs for the hippocampus, mPFC, PCC, and amygdala were constructed using Mask for Region of Interest Analysis (MARINA) software program (Walter et al., 2003; Figure 1). The hippocampal masks were manually sectioned into anterior and posterior ROIs using MRIcron (Rorden, Karnath, \& Bonilha, 2007), based on segmentation guidelines for the hippocampal head and tail, respectively (Malykhin et al., 2007). Although no specific hypotheses were made about the bilateral nature of these ROIs, left and right ROIs were created for each region to investigate the correlated brain activity at a greater level of specificity, which is consistent with previous rsFC studies in PTSD (Sripada et al., 2011; Zhang et al., 2016). 

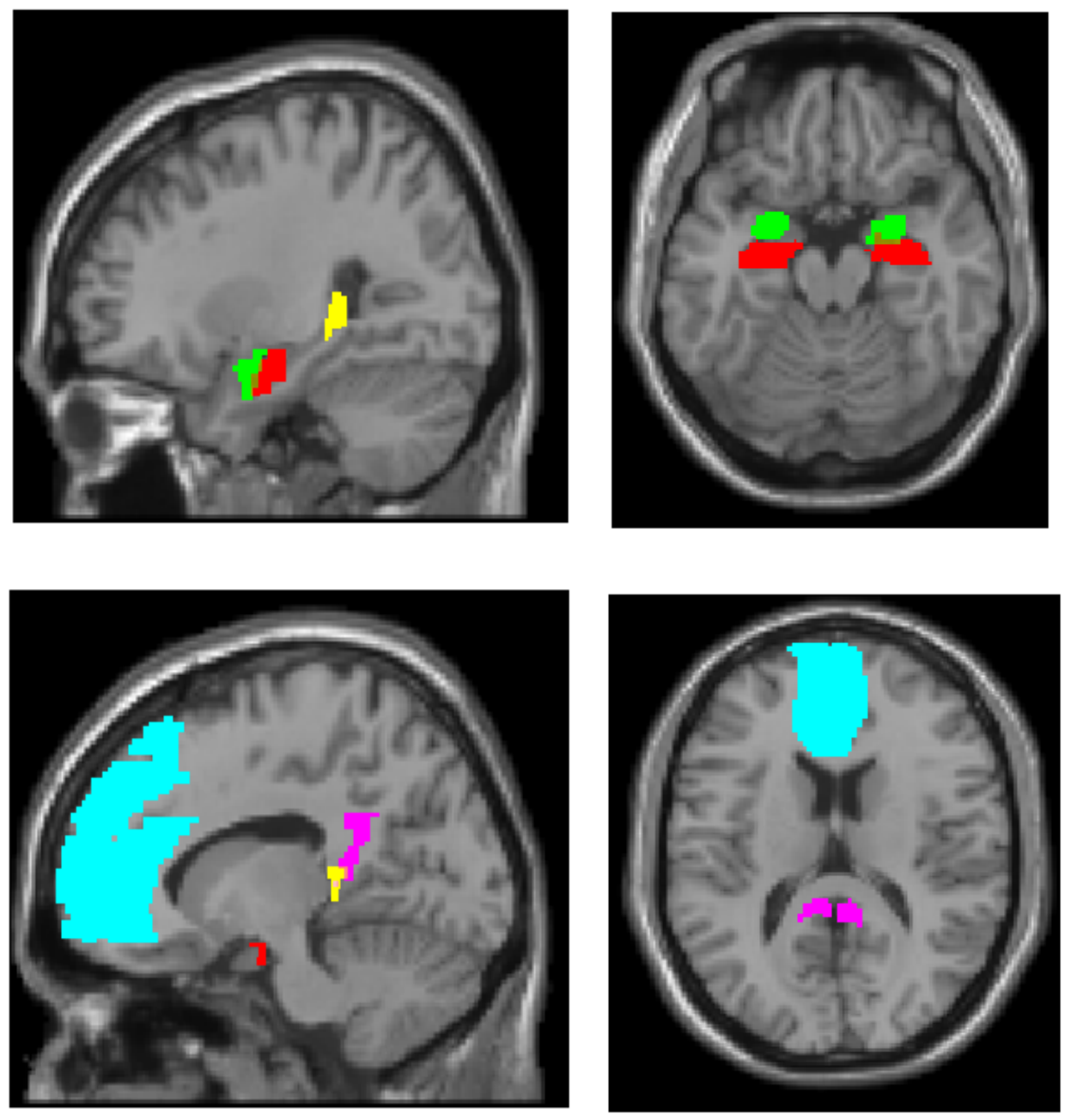

Figure 1. The ROIs used in the present study displayed on a template brain. Red = anterior hippocampus; yellow $=$ posterior hippocampus; green $=$ amygdala; cyan $=\mathrm{mPFC} ;$ magenta $=$ PCC.

\section{Data Analysis}

Spatially averaged time series from the anterior hippocampus, posterior hippocampus, mPFC, PCC, and amygdala were extracted for each participant, which were used to generate FC 
maps between each of the ROIs using a voxel-wise method. The correlation coefficient map was converted into a Fisher's $z$ map using the Fisher's $z$ transformation to improve normality. The $z$ values representing FC between ROIs were transferred to SPSSv.20.0 (IBM Corp., 2011) for group-level contrasts. One-sample t-tests were conducted to examine whether correlated activity between the FC coefficients differ significantly from zero within the PTSD and TEC groups. A threshold of $p<.05$ was used for the group-level contrasts unless otherwise stated. Using t-tests, the FC coefficients that met the significance threshold within groups were then compared between groups to determine whether the hippocampal ROI correlations significantly differ between the PTSD and TEC groups. Comparing only the rsFC that differed from zero for at least one of the groups helps to characterize the ROIs that showed significant rsFC within groups, which is consistent with similar studies (e.g., Sripada et al., 2011). Moreover, this first analysis step narrowed meaningful FCs and minimized the number of group comparisons that were run, which reduced the likelihood of a Type I error. Lastly, FC coefficients were correlated with CAPS scores to determine whether hippocampal rsFC was associated with symptom severity at the time of scan. A subset of the sample from whom a CAPS score was acquired four months post scan $(n=16)$ were used to investigate whether correlated brain activation predicted symptom severity four months post scan within the PTSD and TEC groups separately. 


\section{Results}

\section{ROI Analyses}

One-sample t-tests revealed many of the hippocampal-ROI correlations within groups to differ significantly from zero. The means and standard deviations of the FC coefficients as well as those that differed significantly from zero are summarized in Table 1. Two FC coefficients within the PTSD group differed significantly from zero. In contrast, nine FC coefficients differed significantly from zero within the TEC group. Four of these comparisons survived a Bonferroni correction based on the 48 comparisons that were run. However, all of the significant correlations that met a threshold of $p<.05$ were retained to explore whether these FCs differ between groups at the next level of analysis.

The 11 FC coefficients were compared between groups to determine whether the FC between these regions was significantly different between the PTSD and TEC groups. Correlated activation between the left hippocampal head and the right amygdala was significantly greater for the PTSD group compared to the TEC group, $t(22)=2.218, p=.037, g=0.88$ (see Figure 2). Correlated activation between the left hippocampal tail and the right PCC was significantly greater for the PTSD group compared to the TEC group, $t(22)=-2.149, p=.043, g=0.85$ (see

Figure 2). Lastly, the correlated activation between the left hippocampal tail and the left PCC was significantly greater for the PTSD group compared to the TEC group, $t(22)=-2.182, p=.040, g$ $=0.87$ (see Figure 2). Of note, Levene's test for homogeneity of variances was violated, which may be due to the small sample size. Based on the t-test not assuming homogeneous variances, the aforementioned pattern of differences remained the same and of large effect size, but failed to reach significance $($ all $.10>p>.05)$. 
Table 1

Means and standard deviations for the FC coefficients with significant differences from the oneand two-sample t-tests identified.

\begin{tabular}{|c|c|c|c|c|c|c|c|c|}
\hline \multirow{3}{*}{ Group } & \multirow{3}{*}{ Hippocampus } & & \multicolumn{2}{|l|}{ Amygdala } & \multicolumn{2}{|l|}{ mPFC } & \multicolumn{2}{|l|}{ PCC } \\
\hline & & & Left & Right & Left & Right & Left & Right \\
\hline & & & $\mathrm{M}(\mathrm{SD})$ & $\mathrm{M}(\mathrm{SD})$ & $\mathrm{M}(\mathrm{SD})$ & $\mathrm{M}(\mathrm{SD})$ & $\mathrm{M}(\mathrm{SD})$ & $\mathrm{M}(\mathrm{SD})$ \\
\hline \multirow[t]{4}{*}{ PTSD } & Head & Left & $.98(.62)$ & $.85(.45) \dagger$ & $.60(.93)$ & $.57(1.02)$ & $.42(.78)$ & $.43(.83)$ \\
\hline & & Right & $.62(.50)$ & $.97(.56)$ & $.52(.80)$ & $.51(.79)$ & $.37(.75)$ & $.42(.77)$ \\
\hline & Tail & Left & $.43(.70)$ & $.32(.66)$ & $.61(.94)$ & $.58(.90)$ & $.54(.74)^{*}+$ & $.56(.80)^{*} \dagger$ \\
\hline & & Right & $.39(.75)$ & $.36(.58)$ & $.45(1.04)$ & $.43(.91)$ & $.41(.80)$ & $.46(.89)$ \\
\hline \multirow[t]{4}{*}{ TEC } & Head & Left & $.79(.30)^{* * *}$ & $.53(.24)^{* * * \dagger}$ & $.31(.34)^{* *}$ & $.21(.31)^{*}$ & $.17(.22)^{*}$ & $.14(.23)^{*}$ \\
\hline & & Right & $.45(.31)^{* * *}$ & $.81(.22)^{* * *}$ & $.09(.18)$ & $.04(.19)$ & $-.04(.21)$ & $-.04(.23)$ \\
\hline & Tail & Left & $.05(.24)$ & $.11(.22)$ & $.06(.16)$ & $.02(.19)$ & $.08(.16) \dagger$ & $.07(.18) \dagger$ \\
\hline & & Right & $.08(.24)$ & $.18(.23)^{*}$ & $.07(.22)$ & $.05(.20)$ & $.04(.22)$ & $.10(.21)$ \\
\hline
\end{tabular}

${ }^{*} p<.05,{ }^{*} p<.01,{ }^{* *} p<.001$ for the one-sample t-tests, $\mathrm{rsFC}>0$

$\dagger p<.05$ for the two-sample t-tests, PTSD $>$ TEC

Note. FC values reported in the table are z-transformed $\mathrm{r}$ coefficients. 


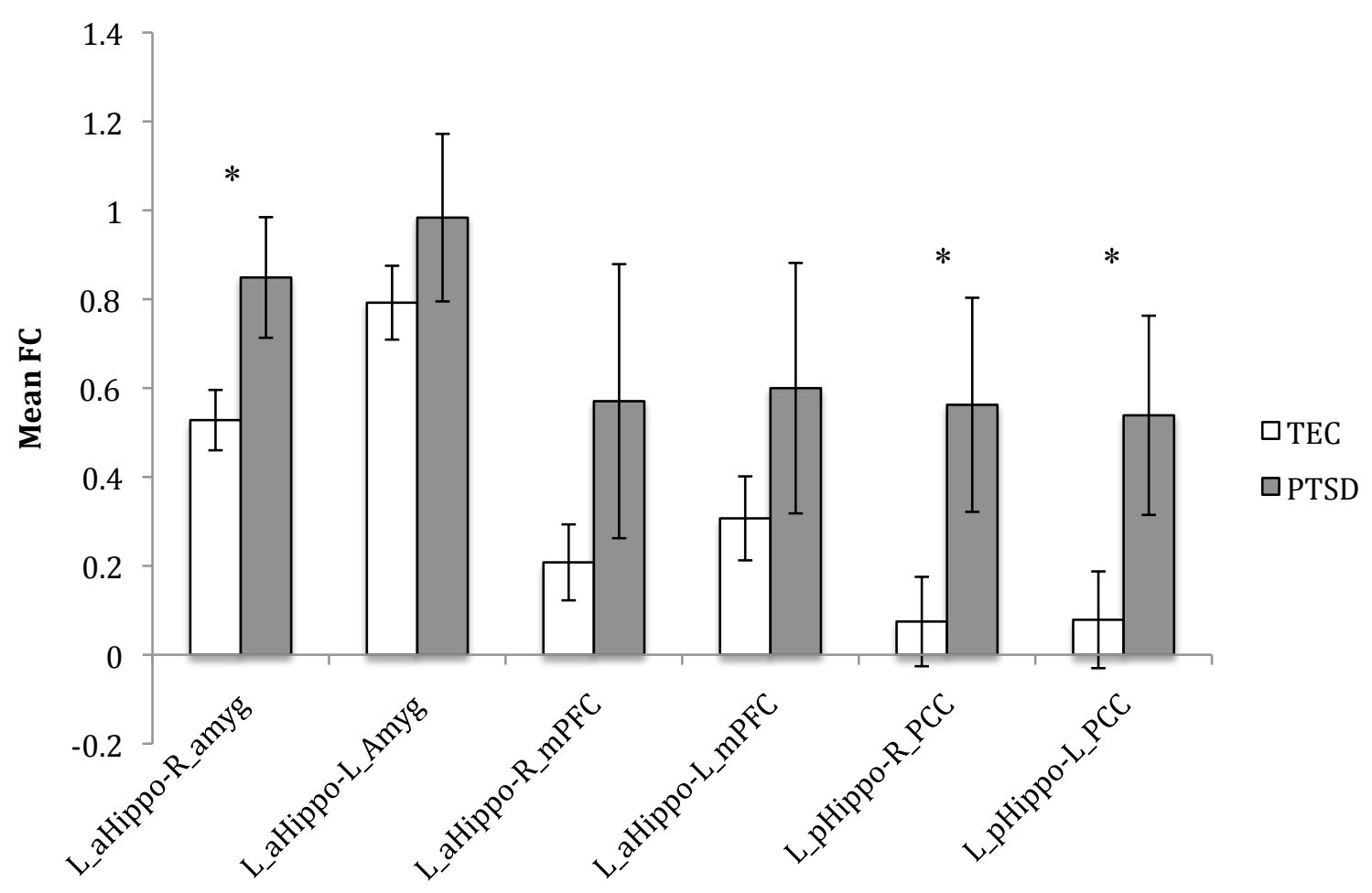

Figure 2. The mean Fisher-z transformed FC values for the PTSD and TEC groups. Displayed are the results for the regions that had been hypothesized to show FC differences between groups with the left side of the hippocampus. Error bars represent standard error.

$* p<.05$

\section{Correlations with CAPS}

CAPS scores at the time of the scan were negatively associated with the FC between the right hippocampal head and the left $(r=-.608, p=.027$, see Figure 3$)$ and right $\mathrm{mPFC}(r=-.689$, $p=.009$, see Figure 4) for the TEC group. These associations were not significant for the PTSD group, all $r$ 's $>-.351, p$ 's $>.200$. Moreover, the FC between the left hippocampal head and the right PCC was negatively associated with CAPS scores for the TEC group at the time of the scan, $r=-.654, p=.015$ (see Figure 5). This association was not significant for the PTSD group, $r=-$ $.384, p=.243$. Upon visual inspection of the correlations (see Figures 3-5) it appears that two participants' FC data may be outliers. Participant five had two FC value z-scores that exceeded 
3.29, which suggests that participant five may be an outlier (Tabachnick \& Fidell, 2007).

Specifically, the FC values that exceeded this threshold were between the right hippocampal head and the right $\mathrm{mPFC}(z=3.35)$ and the right hippocampal head and the left $\mathrm{mPFC}(z=3.51)$.

Excluding participant 5 from the correlation analyses with CAPS at time of scan does not change the results.

CAPS scores four months post scan were negatively associated with the FC between the right hippocampal head and the right amygdala $(r=-.877, p=.001$, see Figure 6$)$ for the TEC group. This association was not significant within the PTSD group, $r=.302, p=.561$. Within the PTSD group, CAPS scores four months post scan were negatively associated with the FC between the left hippocampal head and the left $(r=-.860, p=.028$, see Figure 7) and right PCC, $r=-.886, p=.019$ (see Figure 8). These associations were not significant within the TEC group, all r's $>-.371, p$ 's $>.200$.

Of note, depression scores and time since trauma were not correlated with any of the rsFC reported in previous analyses within both the PTSD and TEC groups. Thus, neither depression nor time since trauma appear to be accounting for the present study findings. 


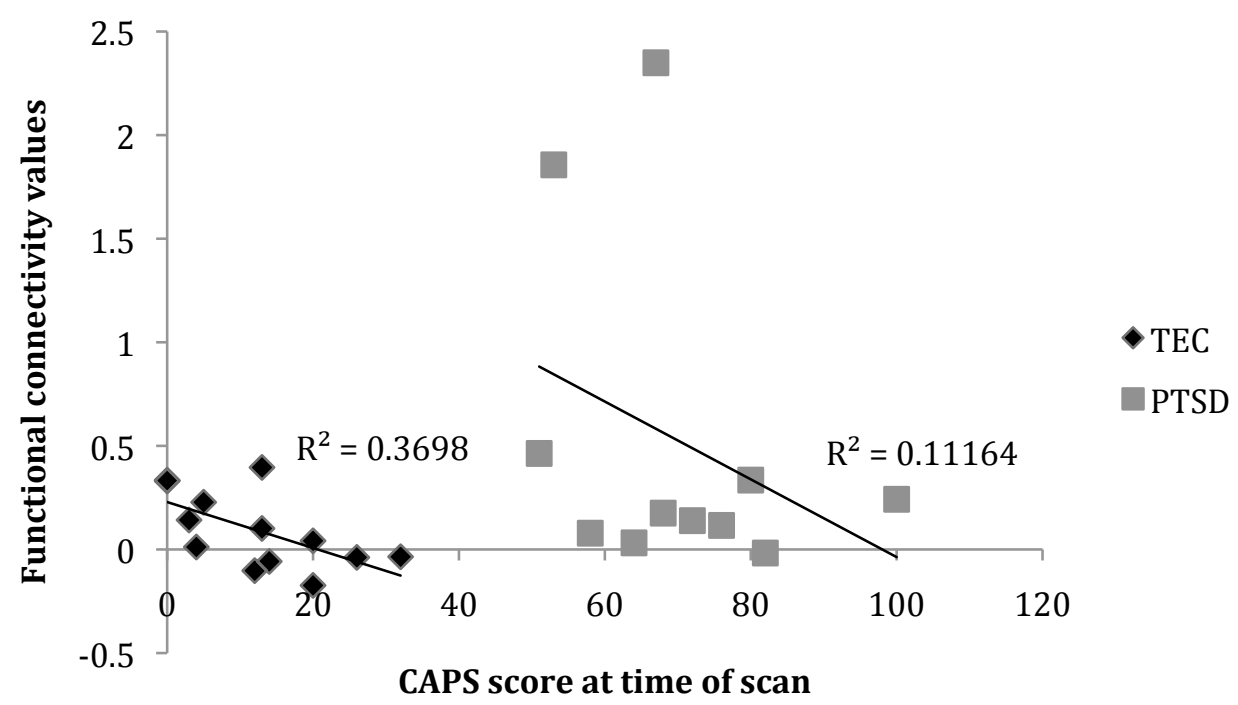

Figure 3. The association between CAPS scores at time of scan and the FC between the right hippocampal head and the left mPFC for the TEC and PTSD groups.

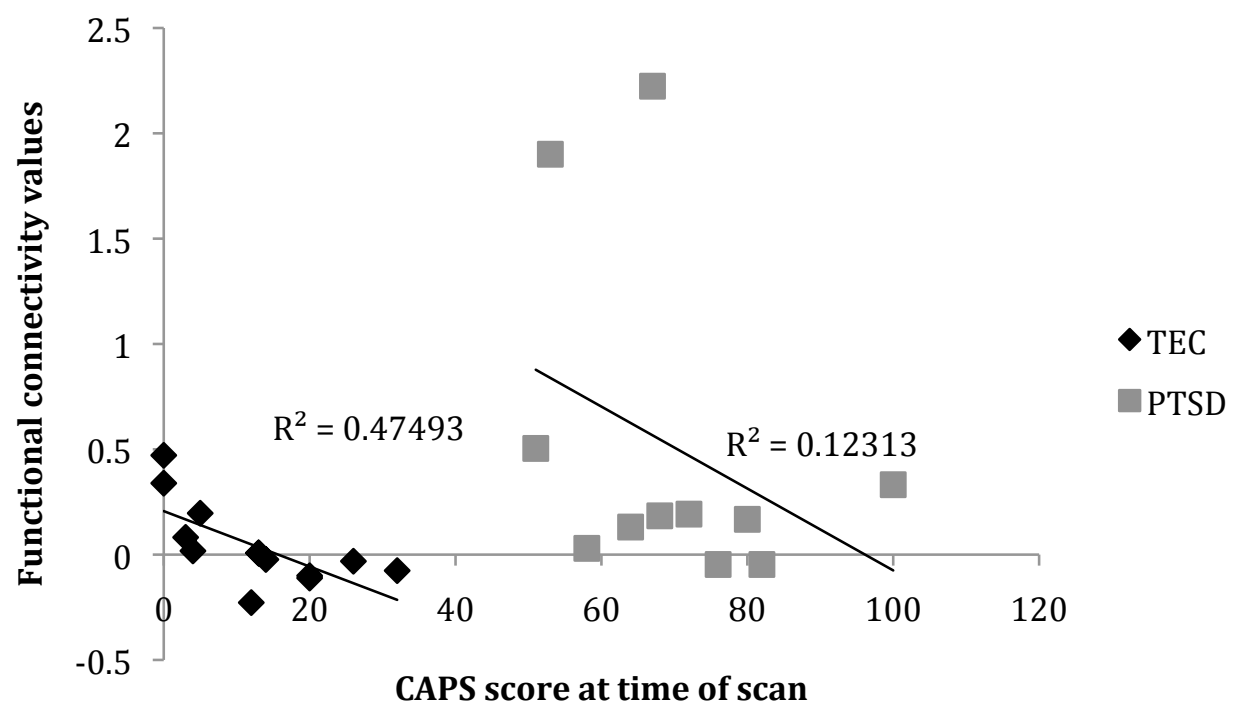

Figure 4. The association between CAPS scores at time of scan and the FC between the right hippocampal head and the right $\mathrm{mPFC}$ for the TEC and PTSD groups. 


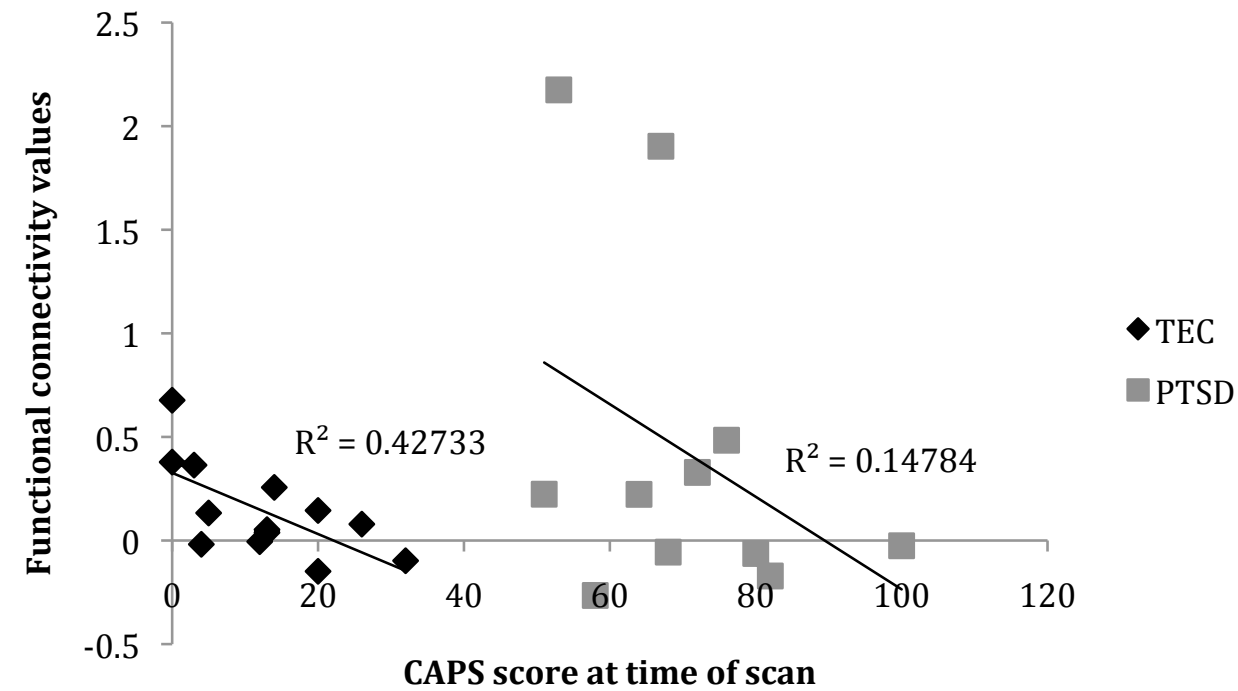

Figure 5. The association between CAPS scores at time of scan and the FC between the left hippocampal head and the right PCC for the TEC and PTSD groups.

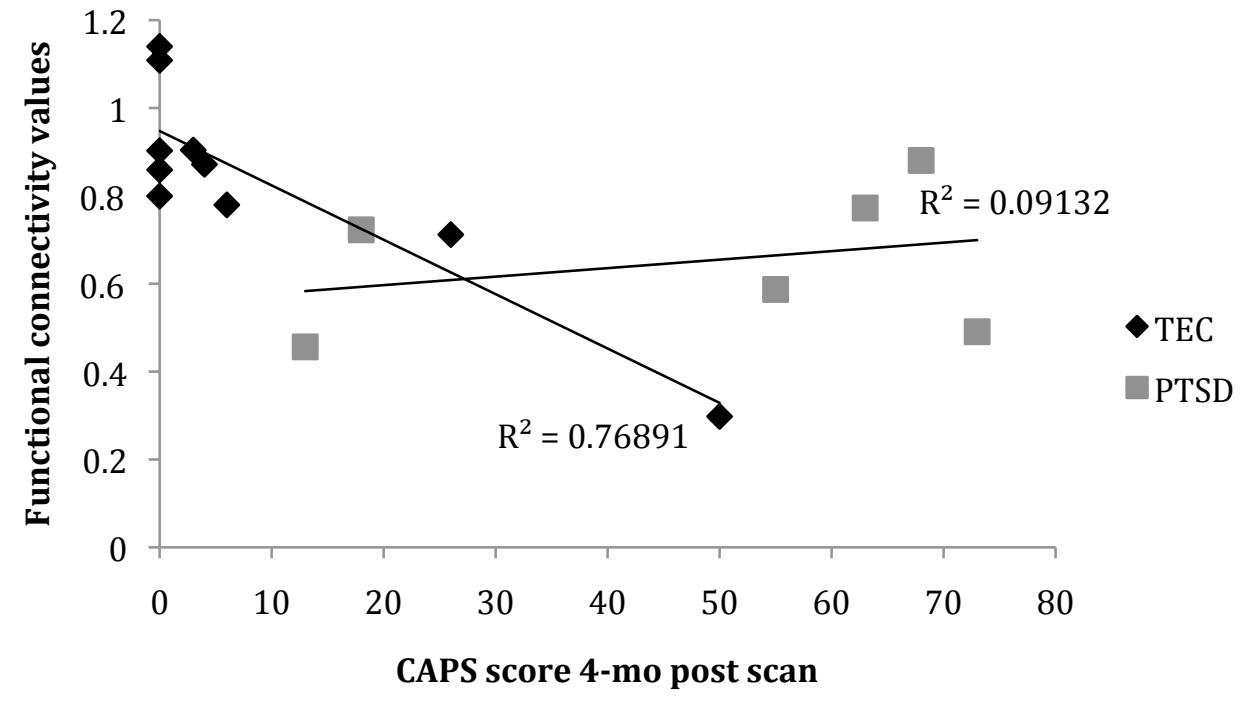

Figure 6. The association between CAPS scores four month post scan and the FC between the right hippocampal head and the right amygdala for the TEC and PTSD groups. 


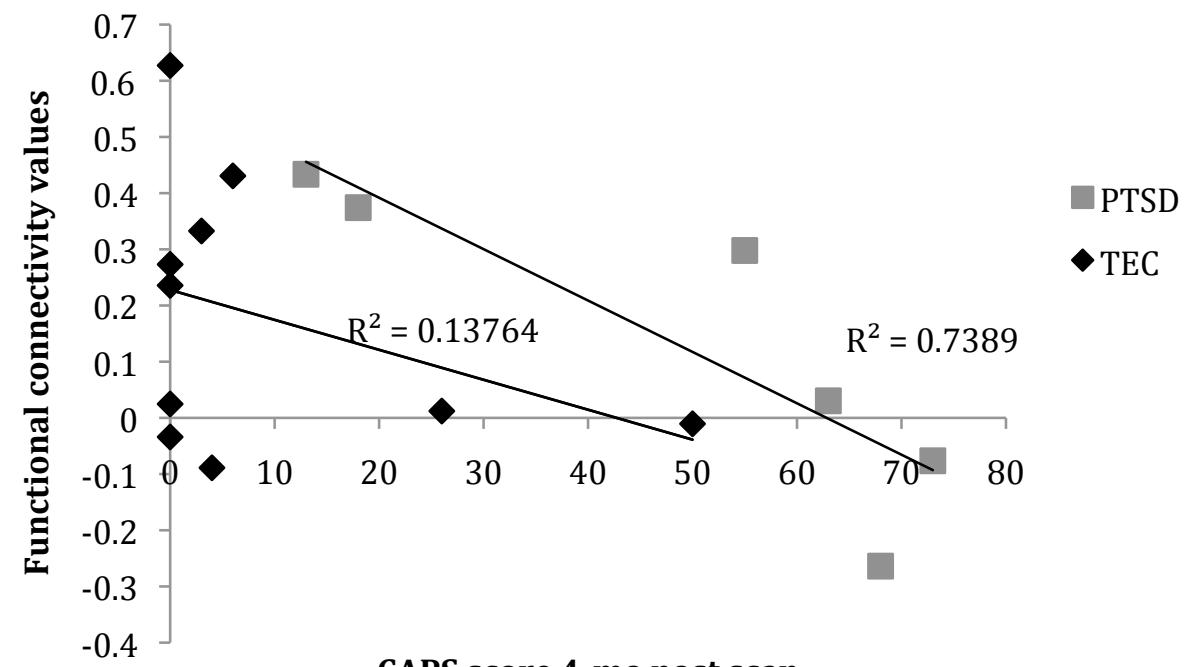

CAPS score 4-mo post scan

Figure 7. The association between CAPS scores four month post scan and the FC between the left hippocampal head and the left PCC for the PTSD and TEC groups.

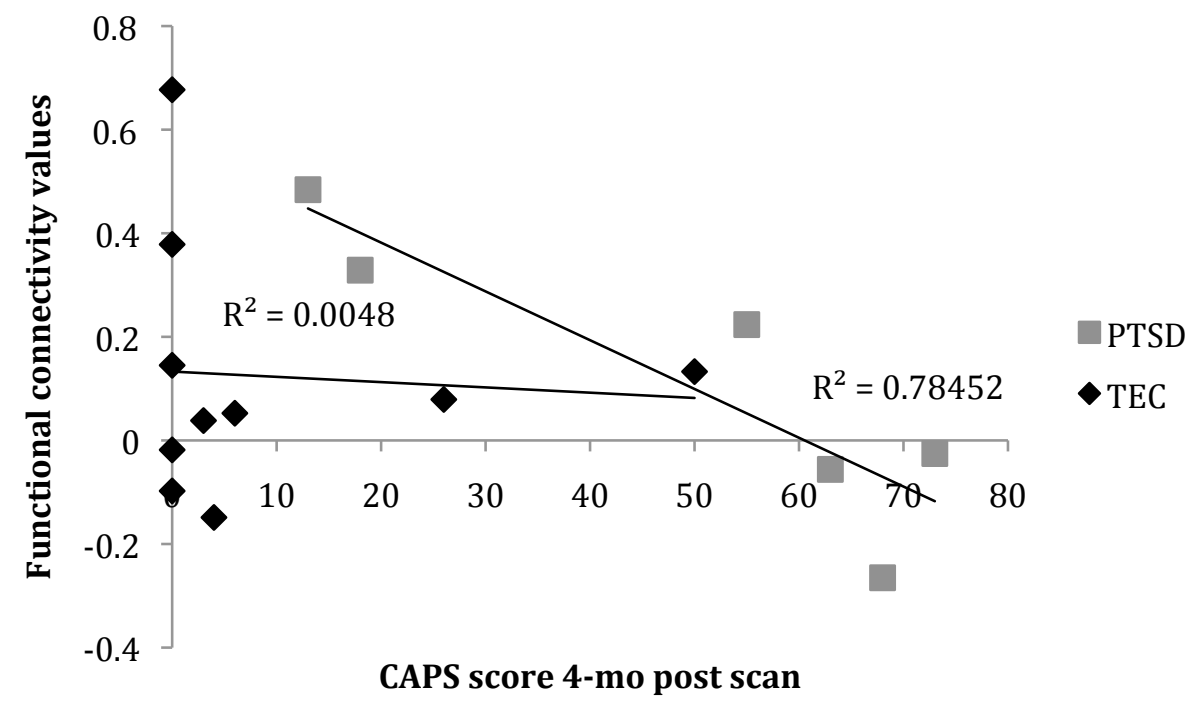

Figure 8. The association between CAPS scores four month post scan and the FC between the left hippocampal head and the right PCC for the PTSD and TEC groups. 


\section{Discussion}

I investigated rsFC between anterior and posterior hippocampal seed regions and brain regions implicated in trauma-related pathophysiology in a sample of PTSD compared to TEC participants. I also investigated the associations between rsFC and PTSD symptom severity at time of scan and four months later. Individuals with PTSD compared to TEC had greater FC between the hippocampus and the amygdala and PCC, but not the mPFC. Moreover, rsFC predicted symptom severity both at the time of scan and four months later in both the PTSD and TEC groups. These findings are discussed in light of the hypotheses and past research below.

\section{H1: The rsFC Between the Anterior Hippocampus and the Amygdala will Differ Between PTSD and TEC}

First, it was hypothesized that the rsFC between the anterior hippocampus and the amygdala would differ between the PTSD and TEC groups. This hypothesis was partially supported; the PTSD group had greater FC between the left anterior hippocampus and the right amygdala compared to the TEC group. The present study used the hippocampus as the seed region as opposed to the amygdala as per previous studies (Rabinak et al. 2011; Sripada et al., 2011; Zhang et al., 2016) in an effort to reconcile the hippocampal-amygdala FC differences in the literature. Moreover, the present study investigated subregional hippocampal FC to refine our understanding of FC between the hippocampus and other ROIs, such as the amygdala. The present study is consistent with the findings of Zhang et al. (2016) supporting greater hippocampal-amygdala FC in PTSD compared to TEC. The present study extends this finding by demonstrating increased FC between the left anterior hippocampus and the right amygdala specifically. Of note, the same pattern of findings was evident for the rsFC between the left anterior hippocampus and the left amygdala as the rsFC for the PTSD group was greater than the TEC group (Figure 2). However, the difference in rsFC did not meet significance, which may be 
attributable to a ceiling effect; these structures had the highest mean FC in both groups (but also substantial variance). Compared to prior studies, the methodology of the present study is the most consistent with Zhang et al. (2016), which may also contribute to the congruence in findings. Specifically, the studies share similar methods of data preprocessing and analysis, both studies used a TEC group, and in both studies the sample consisted of males and females. Of note, enhanced FC between the amygdala and hippocampus has also been found in studies investigating FC in response to emotionally provocative stimuli (Cisler et al., 2014; Jacques, Botzung, Miles, \& Rubin, 2011). Moreover, a meta-analysis from our lab (Patel et al., 2012) and a recent review of rsFC in PTSD (Koch et al., 2016) support hippocampal hyperactivity in PTSD associated with medial-temporal hyperactivity. Thus, the present study findings add to the accumulating evidence supporting attenuated or enhanced activation in the hippocampus and medial-temporal lobe in PTSD (see Hughes \& Shin, 2011 and Patel et al., 2012 for reviews).

Dysfunction between the hippocampus and amygdala is proposed to contribute to key PTSD symptoms including intrusive symptoms, such as nightmares and flashbacks, heightened fear responses, and deficits in contextual processing (Carrion \& Wong, 2012). The present study provides a more refined understanding of hippocampal-amygdala pathophysiology in PTSD as the present study found hyperactive rsFC between the anterior hippocampus in particular and the amygdala compared to the TEC group. This is consistent with human neuroimaging research that supports anterior hippocampal connections with the amygdala (Poppenk et al., 2013). The anterior hippocampus and amygdala activate in response to novel or partially novel stimuli, including faces and environments (Poppenk et al., 2013), which may contribute to the hypervigilence and persistent fear in PTSD. The anterior hippocampus is also preferentially involved in encoding compared to the posterior hippocampus (Schacter \& Wagner, 1999; Spaniol et al., 2009). Moreover, a meta-analysis of fMRI studies supported the role of the anterior 
hippocampus in emotionally-enhanced memory (Murty, Ritchey, Adcock, \& LaBar, 2010). The greater FC between the anterior hippocampus and amygdala in the PTSD group at rest may be associated with stronger encoding of emotionally laden and threatening stimuli when confronted with such stimuli.

\section{H2: The rsFC Between the Anterior Hippocampus and the MPFC will Differ Between PTSD and TEC}

The second hypothesis was that there would be different rsFC between groups for the anterior hippocampus and the mPFC. However, this hypothesis was not supported. Jin et al. (2014) found there to be abnormal connectivity between the mPFC and the hippocampus in a whole-brain FC analysis. Specifically, weaker connectivity was found between the mPFC and the amygdala and hippocampus/parahippocampal gyrus (Jin et al., 2014). Of note, Jin et al. (2014) conducted a whole-brain analysis to investigate the brain regions between which FC differs in PTSD compared to TEC. Moreover, the FC between the hippocampus and the mPFC may have been too subtle in the current study or not present. It is also possible that the prefrontal ROI selected in this study was too liberal. Of note, the FC between the left anterior hippocampus and the bilateral mPFC significantly differed from zero in the TEC group. Although the rsFC was greater in the PTSD group compared to TEC it did not significantly differ from zero or from the TEC group because of the large variance within the PTSD group (see Table 1 and Figure 2). This is a notable negative finding that requires further investigation and replication.

\section{H3: The rsFC Between the Posterior Hippocampus and the PCC will Differ Between PTSD and TEC}

The third hypothesis that there would be group $\mathrm{rsFC}$ differences between the posterior hippocampus and the PCC was supported. Although consistent with past rsFC research that found perturbed FC between the hippocampus and PCC (Bluhm et al., 2009; Chen \& Etkin, 2013), the 
findings are incongruent in the direction of FC. The present study found there to be greater rsFC between the posterior hippocampus and the PCC bilaterally. In contrast, Bluhm et al. (2009) and Chen and Etkin (2013) found there to be reduced FC between the PCC and the hippocampus in PTSD compared to a control group. Moreover, Bluhm et al. (2009) found the reduced FC to be with the right hippocampus whereas the present study found the greater connectivity between the left hippocampus and the bilateral PCC. However, there are several differences between these studies and the present study that may contribute to these discrepancies. Bluhm et al. (2009) and Chen and Etkin (2013) compared FC in PTSD against a healthy control group whereas the present study used a TEC group. Moreover, Bluhm et al. (2009) investigated FC in a female only sample that had been exposed to chronic, early life trauma. The sample characteristics in the present study are more heterogeneous in terms of gender, trauma-type, and time since trauma, which may have contributed to the discrepancy in findings.

The abnormal FC between the posterior hippocampus and PCC is consistent with anatomical research that supports the posterior hippocampus specifically has thalamus-mediated connections to the cingulate gyrus (Poppenk et al., 2013). Given the connections between the posterior hippocampus and other brain regions involved in spatial processing, including the cingulate, precuneus, and visual cortices it has been suggested that the posterior hippocampus plays a role in spatial processing, including spatial memory and navigation (Poppenk et al., 2013). Individuals with PTSD have been found to have impaired performance on visuospatial copying tasks compared to controls (Gurvits, Lasko, Repak, Metzger, Orr, \& Pitman, 2002) and a deficit in their ability to construct a cognitive map of a virtual environment (Tempesta, Mazza, Iaria, De Gennaro, \& Ferrara, 2012). The abnormal FC between the posterior hippocampus and PCC in PTSD compared to TEC may reflect group differences in these processes. More generally, the PCC is part of the default mode network, which plays a role in the integration of 
past events with present stimuli and assessing personal relevance. Group rsFC differences between the hippocampus and PCC may reflect group differences in integration and contextualization of memories, which may contribute to threat hypersensitivity evident in PTSD (Bluhm et al., 2009).

\section{H4: Hippocampal rsFC will be Associated with PTSD Symptom Severity at the Time of Scan and with PTSD Symptom Severity Four Months Post-Scan}

The fourth hypothesis was that hippocampal rsFC would be associated with PTSD symptom severity at the time of scan and would predict PTSD symptom severity four months post-scan. At the time of scan, rsFC was associated with CAPS scores within the TEC group. Specifically, there was a negative association between CAPS scores and connectivity between the right anterior hippocampus and the bilateral $\mathrm{mPFC}$ and the left anterior hippocampus and the right PCC. This indicates that rsFC of the anterior hippocampus is associated with symptom severity in individuals exposed to trauma who did not go on to develop PTSD. Similar trends were observed in the PTSD group; however, the associations were not statistically significant. This may be due to the smaller sample in the PTSD group and less power. It may also be as a result of two high FC scores that increase the distribution of scores making the association less robust within the PTSD group (see Figure 4 for an example). FC in both the PTSD and TEC groups was associated with CAPS scores four months post-scan. In the TEC group, there was a negative association between connectivity of the right anterior hippocampus and the right amygdala and CAPS scores four months post scan. Thus, rsFC of the anterior hippocampus can also predict symptom change in individuals exposed to trauma who did not go on to develop PTSD, which could have implications for understanding whether or not such individuals will develop PTSD. Within the TEC group, one individual had a CAPS score greater than 45 four months post-scan, which is indicative of clinically significant PTSD symptoms. In the PSTD 
group, lower FC between the left anterior hippocampus and the bilateral PCC was associated with higher CAPS scores four months post scan, which suggests poor connectivity between these regions is a risk factor for disease progression. This is the first study to investigate associations between rsFC and PTSD symptoms using the hippocampus as the seed region. Further research is required with larger samples to confirm these associations. Previous research using seed regions such as the PCC (Lanius et al., 2010; Zhou et al., 2012) have found rsFC to be predictive of PTSD symptoms. In addition, Lanius et al. (2010) found the strength of the FC between the PCC and amygdala to predict symptoms 12 weeks post-scan. Collectively, these findings provide support for future investigations of robust imaging biomarkers that predict disease progression.

A meta-analysis suggests there may be distinct changes in the brain between individuals exposed to trauma who did not develop PTSD and individuals never having been exposed to a trauma (Patel et al., 2012). It is suggested that the brain changes in TECs may reflect resilience to developing PTSD (Patel et al., 2012), which is useful when considering biomarkers of the disorder. Thus, the discrepancies in studies investigating rsFC may reflect differences in the research question depending on the control group used in the study. For instance, a study with a healthy control group might address the question: What are the differences in rsFC between individuals who have PTSD and those who have never been exposed to trauma? Whereas a study with a TEC group might address the question: What are the rsFC patterns that distinguish those who go on to develop PTSD versus those who do not following exposure to trauma? The findings of the present study were in accordance with studies that used a similar methodology, and investigated FC differences between individuals with PTSD and those exposed to trauma who did not go on to develop PTSD (e.g., Zhang et al., 2016). Given the different research questions addressed depending on the control group, it is challenging to contrast the finding of studies using difference methods and draw conclusions. This conundrum warrants further consistent 
research that addresses each of the aforementioned questions as they both contribute important information in understanding the pathophysiology underlying PTSD.

\section{A Critical Review of rsFC}

It is also important to address the strengths and limitations of the resting-state imaging approach to study brain networks. Strengths of the resting-state approach have been discussed earlier on in my thesis. Specifically, rsFC is a powerful tool for investigating spontaneous activity of brain networks independently of a task. rsFC avoids the confounds of task-based approaches, which include effort level, differences in approach, task performance, and underlying brain abnormalities that differentiate the groups (Fornito \& Bullmore, 2010; Fox \& Greicius, 2010). rsFC research permits investigation of resting state networks without these difficult to control confounds and provides information on how connectivity patterns vary in different conditions, such as PTSD compared to TEC. However, the meaning of the intrinsic activity remains elusive, which is discussed further below (Cole, Smith, \& Beckmann, 2010). The signal-to-noise ratio in rsFC studies is superior to task-based approaches (Fox \& Greicius, 2010). In task-based fMRI research, only $20 \%$ of the signal is related to the task of interest. Consequently, $80 \%$ of the signal is considered to be noise. In contrast, most of the signals are analyzed in rsFC as the spontaneous activity is of interest (Daliri \& Behroozi, 2013; Fox \& Greicius, 2010). The greater sensitivity in rsFC is useful to recognize imaging abnormalities in individual patients. Within clinical samples, rsFC provides insight into disease biomarkers that could provide diagnostic clarification (Fornito \& Bullmore, 2010; Fox \& Greicius, 2010). A goal of rsFC studies in clinical populations is to use this technology to make meaningful interpretations of rsFC at the level of the patient (Greicius, 2008). For instance, rsFC could be used as an objective initial indicator of treatment response. However, the rsFC literature in PTSD is in the early stages and cannot be used for these clinical purposes at this time. 
A primary criticism of the resting-state approach is that it is unknown what participants are doing when they are told to be 'at rest' (Buckner, Krienen, \& Yeo, 2013; Campbell \& Schacter, 2016; Cole, Smith, \& Beckmann, 2010; Klein, 2014; Morcom \& Fletcher, 2007). Brain activity at rest may be a reflection of the thought processes of the participants at rest, which may vary greatly between participants (Campbell \& Schacter, 2016). There may be differences in the thought process of those with PTSD and TECs at rest that may underlie group differences in $\mathrm{rsFC}$; however, this has yet to be explored. It is suggested that 'at rest' should be viewed as another task state (Campbell \& Schacter, 2016). A second critique of the resting state approach is that the measurement of brain activity is susceptible to motion and physiological artefacts (Campbell \& Schacter, 2016). Artefacts were corrected for in the present study using the DPABI preprocessing toolbox (Yan, Wang, Zuo, \& Zang, 2016); however, it is unknown whether such artefacts can be entirely removed and whether these cleaning processes act as a confound by introducing group differences (Campbell \& Schacter, 2016). Lastly, when relating rsFC to cognition, behaviour, or clinical symptoms, causal inferences cannot be made and thus the order of events is unknown (Cole, Smith, \& Beckmann, 2010).

Thus, as with any approach, there are strengths and limitations to the resting-state approach. Of note, rsFC studies are useful to generate new hypotheses that can be addressed using a task-based approach such as neural networks in relation to the participants' abilities and proficiency. Differences in rsFC between groups in the present study may reflect differences in symptoms and processes, which could be investigated using a task-based approach. For instance, differences in hippocampal-PCC rsFC was suggested to reflect group differences in spatial processing. Spatial processing deficits in PTSD has received little attention in the literature and task-based neuroimaging investigations could be used to explore such deficits further. 
Nevertheless, the present study findings need to be considered within the context of the limitations of the rsFC approach.

\section{Study Strengths}

The present study investigated rsFC of hippocampal subregions in PTSD and is the first to compare rsFC of hippocampal subregions in a sample of PTSD compared to TEC. The hippocampus is not a homogeneous structure and the subregions have independent projections to different brain structures (Poppenk et al., 2013). Thus, to refine our understanding of FC abnormalities in PTSD it is important to consider hippocampal subregions independently. The present study found different FC between the ROIs and the anterior and posterior hippocampus, which underscores the importance of considering hippocampal subregions independently when investigating FC. This study is also the first to investigate how rsFC of the anterior and posterior hippocampus is associated with current and future symptoms, which can allude to disease biomarkers. Another strength of the present study was the use of hypothesis driven ROIs compared to an exploratory analysis as exploratory analyses increase the likelihood of a type I error. Using a seed analysis as the mode of analysis may have biased the results; however, I investigated a priori ROIs that have been found to have abnormal FC with the hippocampus in previous studies. Of note, the present study consisted of a heterogeneous sample as the participants varied in their sex, age, type of trauma exposure, and time since trauma exposure. The bulk of previous research has used very specific samples, such as Chinese earthquake survivors (Zhang et al., 2016), females exposed to early life trauma (Bluhm et al., 2009), car accident victims (Zhou et al., 2012) and veterans (Rabinak et al., 2011; Sripada et al., 2012). The heterogeneity in the present study increases the generalizability of the findings and investigates differences in FC that are common across participants with various trauma exposures and time since trauma. A fruitful direction for future research would be to compare rsFC between PTSD, 
TEC, and non-trauma exposed controls. A study design including all three groups would provide insight into rsFC differences between individuals with PTSD and individuals exposed to trauma who did not go onto develop the disorder compared to trauma naïve participants.

\section{Limitations and Future Directions}

Interpretations of the present study findings should be considered in light of the study's limitations. Although the heterogeneity within the sample increases the generalizability of the findings, the study should be replicated with a larger sample, which would permit moderation analyses to investigate potential differences and commonalities across different trauma types. In addition, the small sample size may have resulted in inability to detect more subtle FC abnormalities due to the low statistical power. The time since trauma exposure varied between participants; thus, the results should be interpreted with caution as changes in the brain can occur over time. In the present study, the rsFC differences between groups and associations with symptoms were not found to be associated with time since trauma. In addition, the nature of the traumatic events varied across groups. It is unclear how hippocampal function may be affected based on different trauma etiologies. It is possible that severity of trauma accounts for changes in rsFC with the hippocampus as opposed to type of trauma. Future research with a larger sample should investigate the association between time since trauma and changes in $\mathrm{rsFC}$ as well as whether type of trauma differentially influences changes in rsFC. However, the type of trauma and time since trauma varied both within the PTSD group and the TEC group and it is valuable information that despite differences in these variables there are common $\mathrm{FC}$ abnormalities that distinguish those with PTSD from TEC.

Consistent with previous studies investigating rsFC in PTSD (e.g., Bluhm et al., 2009; Chen \& Etkin, 2013), some of the participants were using antidepressant medication. It is unclear how taking medication affects results of rsFC studies. However, it is unlikely that the perturbed 
FC of the hippocampus observed in the present study is solely attributable to effects of medication given that the PTSD group also comprised individuals who were not taking any medication. Moreover, individuals in the TEC group were taking antidepressant medication as well. Participants in the PTSD group had comorbidities including anxiety, major depressive disorder, and substance use disorder. It is unknown whether these findings are specific to PTSD or may extend to these other disorders. Of note, the rsFC differences between groups and associations with symptoms were not found to be associated with depression symptoms. This suggests the findings are not accounted for by depressive symptoms. Moreover, compared to the present study, rsFC studies in other disorders have not reported abnormal FC with the hippocampus, such as major depressive disorder (Wang, Hermens, Hickie, \& Lagopoulos, 2012) and anxiety disorders (Brühl, Delsignore, Komossa, \& Weidt, 2014; Mochcovitch, da Rocha Freire, Garcia, \& Nardi, 2014; Peterson, Thome, Frewen, \& Lanius, 2014), which further suggests that the findings in the present study are not accounted for by participant comorbidities. Future neuroimaging research in PTSD is required to investigate the effects of medication use and comorbid symptoms on changes in rsFC. The use of hypothesis-driven ROIs may have constrained the scope of analyses and it would be valuable to explore whole-brain analyses using the hippocampus as the seed region to investigate other ROIs that may have abnormal FC with the hippocampus following trauma exposure. Lastly, to address the primary critique of rsFC research it is suggested that naturalistic viewing paradigms, such as watching a movie, be used to increase compliance and to increase consistency in mental activity across participants (Campbell \& Schacter, 2016). Thus, future investigations of rsFC in PTSD could use a naturalistic viewing paradigm when comparing intrinsic brain activity between groups. 


\section{Implications and Conclusions}

A goal of rsFC research in clinical populations it to understand functional abnormalities underlying the disorder to ultimately establish reliable markers of the disease that can be interpreted in individual patients (Fox \& Greicius, 2010). Such advancements could permit the

prediction of disease progression. However, neuroimaging research in PTSD is not at a stage to achieve this goal. Firstly, the findings are inconsistent, which is likely attributable to different study designs, methods of pre-processing, and methods of analysis. These discrepancies make it challenging to compare studies and draw conclusions. It is unknown how different methodologies relate to one another and there is no theoretical framework to integrate the disparate rsFC findings (Fornito \& Bullmore, 2010). Additional research with comparable methodology is required to acquire a robust understanding of the rsFC differences between PTSD and controls. Furthermore, subsequent research would be required to investigate sensitivity and specificity of biomarkers for differentiating healthy and clinical states (Fox \& Greicius, 2010). Despite that neuroimaging research in PTSD is not at a point where biomarkers can be identified and used to predict disorder features and development at an individual patient level, the present study findings contribute to the rsFC literature in an attempt to move in such a direction. Specifically, the present study investigated rsFC of the anterior and posterior hippocampus with ROIs previously found to have abnormal FC with the hippocampus to enrich our understanding of these FCs in PTSD compared to TEC. Studying hippocampal FC at rest circumvents confounds associated with task-based analyses (Fox \& Greicius, 2010) and permits characterization of abnormal intrinsic hippocampal activity. Thus, the findings provide a more refined understanding of the group differences in hippocampal rsFC and provide support for future investigations of imaging biomarkers predictive of disease progression. 


\section{References}

Admon, R., Milad, M. R., \& Hendler, T. (2013). A causal model of post-traumatic stress disorder: Disentangling predisposed from acquired neural abnormalities. Trends in cognitive sciences, 17(7), 337-347.

American Psychiatric Association. (2013). Diagnostic and statistical manual of mental disorders (5th ed.). Arlington, VA: American Psychiatric Publishing.

Ashburner, J. (2007). A fast diffeomorphic image registration algorithm. NeuroImage, 38(1), 95113.

Astur, R. S., St. Germain, S. A., Tolin, D., Ford, J., Russell, D., \& Stevens, M. (2006). Hippocampus function predicts severity of post-traumatic stress disorder. Cyberpsychology \& Behavior, 9(2), 234-240.

Beck, A. T., Steer, R. A., \& Brown, G. K. (1996). Beck depression inventory-II. San Antonio, 78(2), 490-498.

Behzadi, Y., Restom, K., Liau, J., \& Liu, T. T. (2007). A component based noise correction method (CompCor) for BOLD and perfusion based fMRI. Neuroimage, 37(1), 90-101.

Bonne, O., Brandes, D., Gilboa, A., Gomori, J. M., Shenton, M. E., Pitman, R. K., and Shalev, A. Y. (2001). Longitudinal MRI study of hippocampal volume in trauma survivors with PTSD. Am. J. Psychiatry, 158, 1248-1251.

Bremner, J. D. (2006). Traumatic stress: effects on the brain. Dialogues in clinical neuroscience, 8(4), 445-461.

Bremner, J. D., Scott, T. M., Delaney, R. C., Southwick, S. M., Mason, J. W., Johnson, D. R., . . . Charney, D. S. (1993). Deficits in short-term memory in posttraumatic stress disorder. American Journal of Psychiatry, 150(7), 1015-1019.

Bremner, J. D., Vermetten, E., Afzal, N., \& Vythilingam, M. (2004). Deficits in verbal 
declarative memory function in women with childhood sexual abuse-related posttraumatic stress disorder. The Journal of nervous and mental disease, 192(10), 643-649.

Brewin, C. R., Gregory, J. D., Lipton, M., \& Burgess, N. (2010). Intrusive images in psychological disorders: characteristics, neural mechanisms, and treatment implications. Psychological review, 117(1), 210-232.

Brohawn, K. H., Offringa, R., Pfaff, D. L., Hughes, K. C., \& Shin, L. M. (2010). The neural correlates of emotional memory in posttraumatic stress disorder. Biological psychiatry, 68(11), 1023-1030.

Brühl, A. B., Delsignore, A., Komossa, K., \& Weidt, S. (2014). Neuroimaging in social anxiety disorder - a meta-analytic review resulting in a new neurofunctional model. Neuroscience \& Biobehavioral Reviews, 47, 260-280.

Buckner, R. L., Krienen, F. M., \& Yeo, B. T. T. (2013). Opportunities and limitations of intrinsic functional connectivity MRI. Nature Neuroscience, 16(7), 832-837.

Carrion, V. G., \& Wong, S. S. (2012). Can traumatic stress alter the brain? Understanding the implications of early trauma on brain development and learning. Journal of adolescent health, 51(2), 23-28.

Campbell, K. L., \& Schacter, D. L. (2016). Ageing and the resting state: Is cognition obsolete? Language, Cognition and Neuroscience, 1-8.

Chase, H. W., Clos, M., Dibble, S., Fox, P., Grace, A. A., Phillips, M. L., \& Eickhoff, S. B. (2015). Evidence for an anterior-posterior differentiation in the human hippocampal formation revealed by meta-analytic parcellation of fMRI coordinate maps: Focus on the subiculum. NeuroImage, 113, 44-60.

Chen, A. C., \& Etkin, A. (2013). Hippocampal network connectivity and activation differentiates 
post-traumatic stress disorder from generalized anxiety disorder. Neuropsychopharmacology, 38(10), 1889-1898.

Cisler, J. M., Steele, J. S., Lenow, J. K., Smitherman, S., Everett, B., Messias, E., \& Kilts, C. D. (2014). Functional reorganization of neural networks during repeated exposure to the traumatic memory in posttraumatic stress disorder: an exploratory fMRI study. Journal of psychiatric research, 48(1), 47-55.

Cole, D. M., Smith, S. M., \& Beckmann, C. F. (2010). Advances and pitfalls in the analysis and interpretation of resting-state FMRI data. Frontiers in systems neuroscience, 4(8), 1-15.

Daliri, M., \& Behroozi, M. (2013). Advantages and disadvantages of resting state functional connectivity magnetic resonance imaging for clinical applications. OMICS $J$ Radiology, 3(1), doi:10.4172/2167-7964.1000e123.

Etkin, A., \& Wager, T. D. (2007). Functional neuroimaging of anxiety: A meta-analysis of emotional processing in PTSD, social anxiety disorder, and specific phobia. The American Journal of Psychiatry, 164(10), 1476-1488.

Fanselow, M. S., \& Dong, H. W. (2010). Are the dorsal and ventral hippocampus functionally distinct structures? Neuron, 65(1), 7-19.

First, M. B., Spitzer, R. L., Gibbon, M., \& Williams, J. B. W. (2002). Structured clinical interview for DSM-IV-TR axis I disorders, research version, patient edition with psychotic screen (SCID-I/P W/PSY SCREEN). New York, NY: Biometrics Research.

Fornito, A., \& Bullmore, E. T. (2010). What can spontaneous fluctuations of the blood oxygenation-level-dependent signal tell us about psychiatric disorders?. Current opinion in psychiatry, 23(3), 239-249.

Fox, M. D., \& Greicius, M. (2010). Clinical applications of resting state functional connectivity. Frontiers in systems neuroscience, 4(19), 1-13. 
Fox, M. D., Snyder, A. Z., Vincent, J. L., Corbetta, M., Van Essen, D. C., \& Raichle, M. E. (2005). The human brain is intrinsically organized into dynamic, anticorrelated functional networks. Proceedings of the National Academy of Sciences of the United States of America, 102(27), 9673-9678.

Friston, K. J., Williams, S., Howard, R., Frackowiak, R. S., \& Turner, R. (1996). Movementrelated effects in fMRI time-series. Magnetic Resonance in Medicine, 35(3), 346-355.

Frueh, B. C., Turner, S. M., Beidel, D. C., \& Cahill, S. P. (2001). Assessment of social functioning in combat veterans with PTSD. Aggression and Violent Behavior, 6(1), 7990.

Geuze, E., Vermetten, E., Ruf, M., de Kloet, C. S., \& Westenberg, H. G. (2008). Neural correlates of associative learning and memory in veterans with posttraumatic stress disorder. Journal of psychiatric research, 42(8), 659-669.

Greicius, M. (2008). Resting-state functional connectivity in neuropsychiatric disorders. Current opinion in neurology, 21(4), 424-430.

Gurvits, T. V., Lasko, N. B., Repak, A. L., Metzger, L. J., Orr, S. P., \& Pitman, R. K. (2002). Performance on visuospatial copying tasks in individuals with chronic posttraumatic stress disorder. Psychiatry research, 112(3), 263-268.

Hayes, J. P., LaBar, K. S., McCarthy, G., Selgrade, E., Nasser, J., Dolcos, F., \& Morey, R. A. (2011). Reduced hippocampal and amygdala activity predicts memory distortions for trauma reminders in combat-related PTSD. Journal of psychiatric research, 45(5), 660669.

Hughes, K. C., \& Shin, L. M. (2011). Functional neuroimaging studies of post-traumatic stress disorder. Expert Review of Neurotherapeutics, 11(2), 275-285.

IBM Corp. Released 2011. IBM SPSS Statistics for Windows, Version 20.0. Armonk, NY: IBM 
Corp.

Jacques, P. L. S., Botzung, A., Miles, A., \& Rubin, D. C. (2011). Functional neuroimaging of emotionally intense autobiographical memories in post-traumatic stress disorder. Journal of psychiatric research, 45(5), 630-637.

Jenkinson, M., Bannister, P., Brady, M., \& Smith, S. (2002). Improved optimization for the robust and accurate linear registration and motion correction of brain images. NeuroImage, 17(2), 825-841.

Jin, C., Qi, R., Yin, Y., Hu, X., Duan, L., Xu, Q., ... \& Gong, Q. (2014). Abnormalities in wholebrain functional connectivity observed in treatment-naive post-traumatic stress disorder patients following an earthquake. Psychological Medicine, 44(9), 1927-1936.

Karl, A., Schaefer, M., Malta, L. S., Dörfel, D., Rohleder, N., \& Werner, A. (2006). A metaanalysis of structural brain abnormalities in PTSD. Neuroscience \& Biobehavioral Reviews, 30(7), 1004-1031.

Kennis, M., Rademaker, A. R., van Rooij, S. J. H., Kahn, R. S., \& Geuze, E. (2015). Resting state functional connectivity of the anterior cingulate cortex in veterans with and without posttraumatic stress disorder: Functional connectivity of the ACC in veterans. Human Brain Mapping, 36(1), 99-109.

Klein, C. (2014). The brain at rest: What it is doing and why that matters. Philosophy of Science, 81(5), 974-985.

Koch, S. B., Zuiden, M., Nawijn, L., Frijling, J. L., Veltman, D. J., \& Olff, M. (2016). Aberrant resting-state brain activity in posttraumatic stress disorder: A meta-analysis and systematic review. Depression and anxiety, 33, $592-605$.

Lanius, R. A., Bluhm, R. L., Coupland, N. J., Hegadoren, K. M., Rowe, B., Theberge, J., ... \& Brimson, M. (2010). Default mode network connectivity as a predictor of post-traumatic 
stress disorder symptom severity in acutely traumatized subjects. Acta Psychiatrica Scandinavica, 121(1), 33-40.

Lanius, R. A., Williamson, P. C., Bluhm, R. L., Densmore, M., Boksman, K., Neufeld, R. W., Gati, J. S., and Menon, R. S. (2005). Functional connectivity of dissociative responses in posttraumatic stress disorder: A functional magnetic resonance imaging investigation. Biol. Psychiatry, 57, 873-884.

Li, M., Long, C., \& Yang, L. (2015). Hippocampal-prefrontal circuit and disrupted functional connectivity in psychiatric and neurodegenerative disorders. BioMed Research International, 1-10.

Liberzon, I., \& Sripada, C. S. (2007). The functional neuroanatomy of PTSD: A critical review. Progress in brain research, 167, 151-169.

Malykhin, N. V., Bouchard, T. P., Ogilvie, C. J., Coupland, N. J., Seres, P., \& Camicioli, R. (2007). Three-dimensional volumetric analysis and reconstruction of amygdala and hippocampal head, body and tail. Psychiatry Research: Neuroimaging, 155(2), 155-165.

Marin, M. F., Song, H., VanElzakker, M. B., Staples-Bradley, L. K., Linnman, C., Pace-Schott, E. F., ... \& Milad, M. R. (2016). Association of Resting Metabolism in the Fear Neural Network With Extinction Recall Activations and Clinical Measures in Trauma-Exposed Individuals. American Journal of Psychiatry. http://dx.doi.org/10.1176/appi.ajp.2015.14111460

Marshall, R. D., Olfson, M., Hellman, F., Blanco, C., Guardino, M., \& Struening, E. L. (2001). Comorbidity, impairment, and suicidality in subthreshold PTSD. The American Journal of Psychiatry, 158(9), 1467-1473.

Menon, V. (2011). Large-scale brain networks and psychopathology: a unifying triple network model. Trends in cognitive sciences, 15(10), 483-506. 
Mochcovitch, M. D., da Rocha Freire, R. C., Garcia, R. F., \& Nardi, A. E. (2014). A systematic review of fMRI studies in generalized anxiety disorder: evaluating its neural and cognitive basis. Journal of affective disorders, 167, 336-342.

Moores, K. A., Clark, C. R., McFarlane, A. C., Brown, G. C., Puce, A., \& Taylor, D. J. (2008). Abnormal recruitment of working memory updating networks during maintenance of trauma-neutral information in post-traumatic stress disorder. Psychiatry Research: Neuroimaging, 163(2), 156-170.

Morcom, A. M., \& Fletcher, P. C. (2007). Does the brain have a baseline? Why we should be resisting a rest. NeuroImage, 37(4), 1073-1082.

Murty, V. P., Ritchey, M., Adcock, R. A., \& LaBar, K. S. (2010). fMRI studies of successful emotional memory encoding: A quantitative meta-analysis. Neuropsychologia, 48(12), 3459-3469.

Nadel, L., Hoscheidt, S., \& Ryan, L. R. (2013). Spatial cognition and the hippocampus: The Anterior-Posterior axis. Journal of Cognitive Neuroscience, 25(1), 22-28.

Patel, R., Spreng, R.N., Shin, L.M., Girard, T.A. (2012). Neurocircuitry models of posttraumatic stress disorder and beyond: a meta-analysis of functional neuroimaging studies. Neurosci. Biobehav. Rev., 36(9), 2130-2142.

Peterson, A., Thome, J., Frewen, P., \& Lanius, R. A. (2014). Resting-state neuroimaging studies: A new way of identifying differences and similarities among the anxiety disorders?. The Canadian Journal of Psychiatry, 59(6), 294-300.

Poppenk, J., Evensmoen, H. R., Moscovitch, M., \& Nadel, L. (2013). Long-axis specialization of the human hippocampus. Trends in Cognitive Sciences, 17(5), 230.

Poppenk, J., \& Moscovitch, M. (2011). A hippocampal marker of recollection memory ability among healthy young adults: contributions of posterior and anterior 
segments. Neuron, 72(6), 931-937.

Rabinak, C. A., Angstadt, M., Welsh, R. C., Kenndy, A. E., Lyubkin, M., Martis, B., \& Phan, K. L. (2011). Altered amygdala resting-state functional connectivity in post-traumatic stress disorder. Frontiers in Psychiatry, 2(62), 1-8.

Rauch, S. L., Shin, L. M., \& Phelps, E. A. (2006). Neurocircuitry models of posttraumatic stress disorder and extinction: Human neuroimaging Research—Past, present, and future. Biological Psychiatry, 60(4), 376-382.

Rauch, S. L., Shin, L. M., Segal, E., Pitman, R. K., Carson, M. A., McMullin, K., ... \& Makris, N. (2003). Selectively reduced regional cortical volumes in post-traumatic stress disorder. Neuroreport, 14(7), 913-916.

Rauch, S. L., Shin, L. M., Whalen, P. J., \& Pitman, R. K. (1998). Neuroimaging and the neuroanatomy of PTSD. CNS Spectrums, 3, 30-41.

Rorden, C., Karnath, H.-O., \& Bonilha, L. (2007). Improving lesion-symptom mapping. Journal of Cognitive Neuroscience, 19, 1081-1088.

Roca, V., \& Freeman, T. W. (2001). Complaints of impaired memory in veterans with PTSD. American Journal of Psychiatry, 158(10), 1738-1739.

Shin, L. M., \& Liberzon, I. (2010). The neurocircuitry of fear, stress, and anxiety disorders. Neuropsychopharmacology, 35(1), 169-191.

Shin, L. M., Shin, P. S., Heckers, S., Krangel, T. S., Macklin, M. L., Orr, S. P., Lasko, N., Segal, E., Makris, N., Richert, K., Levering, J., Schacter, D. L., Alpert, N. M., Fischman, A. J., Pitman, R. K., \& Rauch, S. L. (2004). Hippocampal function in posttraumatic stress disorder. Hippocampus, 14, 292-300.

Solomon, Z. (1989). PTSD and social functioning. Social Psychiatry and Psychiatric Epidemiology, 24(3), 127-133. 
Song, X. W., Dong, Z. Y., Long, X. Y., Li, S. F., Zuo, X. N., Zhu, C. Z., He, Y., Yan, C. G., \& Zang, Y. F. (2011). REST: a toolkit for resting- state functional magnetic resonance imaging data processing. PLoS ONE, 6(9).

Spaniol, J., Davidson, P. S., Kim, A. S., Han, H., Moscovitch, M., \& Grady, C. L. (2009). Eventrelated fMRI studies of episodic encoding and retrieval: meta-analyses using activation likelihood estimation. Neuropsychologia, 47(8), 1765-1779.

Sripada, R. K., King, A. P., Garfinkel, S. N., Wang, X., Sripada, C. S., Welsh, R. C., \& Liberzon, I. (2012). Altered resting-state amygdala functional connectivity in men with posttraumatic stress disorder. Journal of Psychiatry \& Neuroscience: JPN, 37(4), 241249.

Tabachnick, B. G., \& Fidell, L. S. (2007). Using multivariate statistics $\left(5^{\text {th }}\right.$ ed.) Pearson/Allyn \& Bacon.

Tempesta, D., Mazza, M., Iaria, G., De Gennaro, L., \& Ferrara, M. (2012). A specific deficit in spatial memory acquisition in post-traumatic stress disorder and the role of sleep in its consolidation. Hippocampus, 22(5), 1154-1163.

Thomaes, K., Dorrepaal, E., Draijer, N. P., de Ruiter, M. B., Elzinga, B. M., van Balkom, A. J., ... \& Veltman, D. J. (2009). Increased activation of the left hippocampus region in Complex PTSD during encoding and recognition of emotional words: A pilot study. Psychiatry Research: Neuroimaging, 171(1), 44-53.

Uddo, M., Vasterling, J. J., Brailey, K., \& Sutker, P. B. (1993). Memory and attention in combatrelated post-traumatic stress disorder (PTSD). Journal of Psychopathology and Behavioral Assessment, 15(1), 43-52.

van den Heuvel, Martijn P, \& Hulshoff Pol, H. E. (2010). Exploring the brain network: A review on resting-state fMRI functional connectivity. European 
Neuropsychopharmacology, 20(8), 519-534.

Vasterling, J. J., Duke, L. M., Brailey, K., Constans, J. I., Allain, A. N., \& Sutker, P. B. (2002). Attention, learning, and memory performances and intellectual resources in Vietnam veterans: PTSD and no disorder comparisons. Neuropsychology, 16(1), 5-14.

Walter, B., Blecker, C., Kirsch, P., Sammer, G., Schienle, A., Stark, R., \& Vaitl, D. (2003, June). MARINA: An easy to use tool for the creation of MAsks for Region of INterest Analyses. In 9th International Conference on Functional Mapping of the Human Brain (Vol. 19).

Wang, L., Hermens, D. F., Hickie, I. B., \& Lagopoulos, J. (2012). A systematic review of restingstate functional-MRI studies in major depression. Journal of affective disorders, 142(1), $6-12$.

Werner, N. S., Meindl, T., Engel, R. R., Rosner, R., Riedel, M., Reiser, M., and Fast, K. (2009). Hippocampal function during associative learning in patients with posttraumatic stress disorder. J. Psychiatr. Res., 43, 309-318.

Yan, C. G., Wang, X. D., Zuo, X. N., \& Zang, Y. F. (2016). DPABI: Data Processing \& Analysis for (Resting-State) Brain Imaging. Neuroinformatics, 1-13.

Yehuda, R., Keefe, R. S., Harvey, P. D., \& Levengood, R. A. (1995). Learning and memory in combat veterans with posttraumatic stress disorder. The American journal of psychiatry, 152(1), 137-139.

Yin, Y., Jin, H., Jin, C., Hu, X., Duan, L., Li, Z. ... Gong, Q. (2011). Altered resting-state functional connectivity of thalamus in earthquake-induced posttraumatic stress disorder: A functional magnetic resonance imaging study. Brain Research, 1411, 98-107. Zhang, X., Zhang, J., Wang, L., Li, R., \& Zhang, W. (2016). Altered resting-state functional connectivity of the amygdala in Chinese earthquake survivors. Progress in NeuroPsychopharmacology \& Biological Psychiatry, 65, 208-214. 
Zhou, Y., Wang, Z., Qin, L., Wan, J., Sun, Y., Su, S.. ... Xu, J. (2012). Early altered resting-state functional connectivity predicts the severity of post-traumatic stress disorder symptoms in acutely traumatized subjects. PloS One, 7(10), 1-6. 